\title{
SULLA NATURA DELLE CAUSE CHE GENERANO I MICROSISMI
}

\author{
M. GIORgI - E. Rosini $\left({ }^{*}\right)$
}

1. Premessa. - Il presente studio fa parte di una ricerca sistematica sui microsismi in relazione alle perturbazioni atmosferiche intrapresa dall'Istituto Nazionale di Geofisica in collaborazione col Servizio Meteorologico dell'Aeronautica Italiana.

Sotto il nome di microsismi i sismologi generalmente comprendono tutti i movimenti del suolo rilevabili con $i$ sismografi e dovuti alle cause più diverse e aventi aspetti caratteristici a seconda della loro origine perché con questa varia la natura delle onde elastiche ad essi associata (microsismi dovuti al vento, al traffico, ad azioni meccaniche sul suolo in zone industriali, alle onde marine, ai marosi sulle coste rocciose, alla pioggia $e$ in genere alle grandi perturbazioni meteorologiche).

In questo studio con la parola " microsismi » o " agitazione microsismica » intendiamo riferirci soltanto a quella oscillazione del suolo a carattere permanente, di periodo ed ampiezza più o meno regolare, di cui è ormai accertata la intima correlazione con le perturbazioni atmosferiche.

In alcuni nostri precedenti lavori $\left({ }^{(1.2)}\right.$ abbiamo indicato $i$ principali problemi che ancora rimangono insoluti nel fenomeno dei microsismi. Da molti decenni essi hanno costituito l'appassionato oggetto di una pletorica serie di memorie in massima parte dovute a studiosi di sismologia; le pubblicazioni degli ultimi decenni riguardanti i microsismi assommano a diverse centinaia $\left({ }^{3}\right)$. I più eminenti geofisici dei diversi paesi si sono occupati dei microsismi nelle indagini più disparate.

Il fondamentale problema dei microsismi attorno al quale si sono appuntati principalmente gli sforzi dei ricercatori è stato quello della ricerca della causa diretta che genera i microsismi stessi.

Fu molto facile già ai primi osservatori di constatare che i mi-

${ }^{*}$ ) Questa nota costituisce uno sviluppo largamente documentato di una ricerca eseguita dagli Autori i cui risultati furono oggetto di una comunicazione alla XLII Riunione della Soc. It. per il Progresso delle Scienze tenuta a Roma il 28 novembre 1949. 
crosismi il più delle volte non dipendono da cause connesse a particolari condizioni meteorologiche perturbate locali; così, per citare soltanto alcune tappe nella storia degli studi microsismici, De Rossi ( ${ }^{4}$ ) aveva già trovato nel 1874 una certa relazione tra i movimenti microsismici e le variazioni barometriche; successivamente J. Algué ( ${ }^{5}$ ) nel 1900 mise già in evidenza la relazione tra la attività microsismica registrata nell'Osservatorio di Manila e la esistenza e posizione di cicloni tropicali. Wiechert $\left({ }^{6}\right)$ nel 1905 espose la sua ipotesi, che la scuola tedesca $\left({ }^{7}\right)$ sostenne e trova tuttora sostenitori, che $\mathrm{i}$ microsismi sono dovuti all'urto dei marosi contro coste ripide e rocciose; ma Benndorf $\left(^{8}\right)$ nel 1911 trovò che condizione necessaria ma non sempre sufficiente per il verificarsi di certe tempeste microsismiche in Europa è la esistenza di un marcato centro depressionario sull'Oceano Atlantico; d'altra parte Hecker $\left({ }^{9}\right)$ nel 1914 aveva mostrato il concorde andamento simultaneo dell'agitazione microsismica registrata in un certo numero di stazioni europee. I lavori di Gherzi ( $\left.{ }^{10}\right)$ nel 1932 segnano un ulteriore passo verso la risoluzione del problema della origine dei microsismi; essi illustrano la stretta connessione tra l'andamento dei microsismi e la traiettoria dei cicloni tropicali in mare aperto. Banerji (1935) ritiene inoltre che la pressione delle onde del mare può trasmettersi al fondo dell'oceano dando luogo a vibrazioni forzate della crosta solida che costituiscono i microsismi (11). Ramirez (1938) pensò di risolvere il problema della individuazione della direzione di provenienza dei microsismi, per mezzo di una stazione tripartita; egli arrivò alla conclusione che detta origine era in mare aperto sull'oceano in corrispondenza di forti centri depressionari $\left({ }^{12}\right)$; così pure le ricerche condotte da Gilmore (13) ed altri mediante l'ausilio di stazioni tripartite in America e nel Pacifico hanno messo in evidenza sempre meglio la stretta dipendenza tra l'attività microsismica e i cicloni extra-tropicali e tropicali (uragani o tifoni) e sopratutto che l'intensità microsismica è in relazione col movimento del centro ciclonico sull'oceano e decresce notevolmente quando il ciclone passa dal mare sul continente; ancora più recentemente (1948) lavori di Don I,eet $\left({ }^{14}\right)$, di Jones $\left({ }^{15}\right)$, e nostri $\left({ }^{1.2}\right)$ mettevano in evidenza la stretta dipendenza della agitazione microsismica registrata in certi Osservatori e alcune perturbazioni frontali.

Il problema della individuazione della causa o delle cause che generano i microsismi ̀̀ stato tuttavia sempre impostato in maniera troppo unilaterale e prevalentemente hasato sulla misura di alcune correlazioni statistiche tra agenti meteorologici e attività microsismi- 
ca. Per lo più i ricercatori si sono soffermati ad esaminare una particolare causa generalmente trascurandone altre concomitanti. La connessione tra microsismi e fattori legati alle situazioni meteorologiche, come si è detto avanti, fu notata fin dalle prime osservazioni ma in maniera molto grossolana; il comportamento di tutti gli elementi legati a dette situazioni furono confrontati con l'attività microsismica ma quasi singolarmente non in un unico complesso unitario quale è quello da essi costituito e che solo determina il carattere delle situazioni stesse: i marosi sulle coste, lo stato del mare, la pressione, il vento, i centri depressionari, le perturbazioni frontali, ecc., hanno costituito elementi separati con i quali non è stato difficile ai vari ricercatori trovare una documentazione che avallasse le loro affermazioni circa la causa dei microsismi; ed anzi era impossibile non trovare correlazioni statistiche che non provassero le diverse ipotesi; detti elementi sono in genere concomitanti e non è facile evidentemente una discriminazione fra essi. Tuttavia questi numerosi studi anche se non condotti sempre con la necessaria razionalità scientifica hanno consentito di focalizzare sempre meglio il problema.

È da notare inoltre che la questione è stata per lo più affrontata da sismologi i quali l'hanno sviscerata abbastanza esaurientemente dal punto di vista sismico, con dettagli circostanziati circa la natura delle onde elastiche, l'andamento dei periodi e delle ampienze, l'influenza degli strati terrestri, il comportamento nella propagazione, ecc., ma dal punto di vista meteorologico non ha avuto una proporzionata attenzione.

È da osservare dunque che gli elementi meteorologici si compongono a formare la vicenda meteorologica, che è un insieme molto complesso di essi. Gli elementi infatti da soli perdono ogni significato o per lo meno gran parte, in quanto non costituiscono altro che un aspetto o un componente della situazione, mentre la loro azione concomitante e combinata li fa interdipendenti e non possono quindi essere considerati staccati. Per modo che detti elementi, sempre combinati e contemporanei, separatamente messi in relazione con l'attività microsismica non avrebbero mai potuto costituire una prova definitiva come in realtà si è constatato trovandosi tuttora fautori dell'una o dell'altra causa.

Partendo da queste considerazioni, come del resto è stato già da nrsi indicato in precedenti lavori, abbiamo constatato che la sola via possibile da intraprendere per risolvere in maniera decisiva il problema della individuazione della causa dei microsismi era quella di eseguire un esame minuzioso e approfondito delle situazioni meteo- 
rologiclie tenendo conto di tutto il complesso degli elementi che le determinano confrontate con la contemporanea attività microsismica. Dovevamo quindi avvalerci dei moderni mezzi dell'indagine meteorologica sia dal punto di vista delle relative acquisizioni scientifiche più recenti, sia da quello della organizzazione odierna dei servizi meteorologici per il rilievo e lo studio sistematico delle situazioni. Era quindi per tale opera necessaria la collaborazione più stretta tra il sismologo ed il meteorologo. A tale scopo per quanto riguarda il lato sismologico della questione abbiamo potuto avvalerci di tutte le necessarie prestazioni e del materiale di osservazione dell'osservatorio Centrale di Roma dell'Istituto Nazionale di Geofisica, e per quanto riguarda invece la parte meteorologica, che in questo caso era di somma importanza in quanto avreble dovuto fornirci un'esauriente risposta al nostro problema, abbiamo potuto usufruire nella maniera più ampia ed agevole di tutta la organizzazione e la documentazione del Servizio Meteorologico dell'Aeronantica Italiana e quindi attraverso esso dei dati e delle elaborazioni dei servizi meteorologici degli altri Paesi.

Posto così il prohlema ci si è presentata ancora la necessità di ridurlo a limiti più circostanziati. Come è stato esposto in una nostra nota già citata $\left({ }^{1}\right)$, l'agitazione microsismica presenta caratteristiche diverse a seconda del luogo e della distanza d'onde proviene: a prescindere dall'ampiezza, dalla quale si può dedurre una valutazione dell'intensità microsismica in relazione con l'attività, ubicazione ed estensione della presunta causa da cui l'agitazione microsismica stessa prende origine e che d'altra parte dipende pure dalla natura geologica e dallo spessore degli strati terrestri interessati, il periodo della oscillazione costituisce una caratteristica fondamentale dei microsismi ed è in intima relazione con le cause dei microsismi stessi e con il luogo dove esse agiscono.

Riassumiamo qui i risultati delle indagini già condotte riguardanti i periodi della agitazione microsismica registrata a Roma in relazione al luogo ove agisce la presunta causa che la genera.

1,5-2,5 sec. - microsismi di origine locale provenienti ciò̀ dal Tirreno prospicente fino alla Sardegna;

3-3,5 sec. - microsismi aventi origine nel Golfo di Genova e nel Mar Iigure;

3.4 sec. - microsismi provenienti dal Tirreno centro-meridionale:

4-5 sec. - microsismi aventi origine nel Mediterraneo occiden- 
tale tra le Baleari e la Sardegna o nello Jonio o nel Mediterraneo centrale;

6-10 sec. - microsismi di origine atlantica.

Il periodo della oscillazione per ogni tempesta microsismica può variare per due fattori fondamentali: può aumentare cioè sia con l'allontanamento della causa agente sia con l'aumentare della intensità (ampiezza) dell'agitazione.

Si può notare ancora che $\mathrm{i}$ microsismi hanno un aspetto piuttosto regolare quando provengono da una sorgente unica. Spesso le cause agenti, pur della stessa natura, sono molteplici e quindi i microsismi provengono da luoghi diversi, si sovrappongono e assumono un aspetto intricato e non sempre $\dot{e}$ facile individuare le fasi componenti.

Per metterci nelle condizioni che ci offrissero la magriore probabilità di successo, abbiamo pensato di focalizzare il settore per il quale avevamo la maggiore abbondanza di dati meteorologici diretti o di elaborazione: cioè il Mediterraneo; successivamente abbiamo ancora ulteriormente limitato la nostra indagine alle tempeste microsismiche di breve periodo (da $4 \mathrm{sec}$ in giù) di un intero anno (1949) e quindi connesse con perturbazioni di origine prevalentemente tirrenica.

Nel presente studio si è seguito il criterio che ci sembra meglio resistente ad ogni critica, non di scergliere esempi singoli indiscutibilmente favorevoli ad una determinata tesi, ma di procedere allo studio parallelo del comportamento dell'attività microsismica e della evoluzione contemporanea delle vicende meteorologiche con continuità per tutto un anno di osservazioni. In tal modo ci è stato possibile pervenire ad un risultato oggettivo derivante dalla ricerca e non conseguenza di una ipotesi ad essa aprioristicamente preposta.

(uesto risultato che è largamente provato dalla documentazione più sotto illustrata, è grià stato da noi esposto in una comunicazione al XLII Congresso Nazionale della S.I.P.S. tenuto a Roma il 28 novembre $1949\left({ }^{16}\right)$ e può cosi riassumersi : $i$ microsismi esaminati aventi, come si è detto, origine sul Mediterraneo centro-occidentale vengono generati nelle zone interessate da nuclei di tendenze barometriche positive, stazionari $o$ in movimento.

La documentazione completa, per ovvie ragioni, è stata limitata soltanto ad alcune tempeste microsismiche.

2. Metodi e mezzi adoperati. - Per quanto concerne i rilievi sismici, praticamente abbiamo proceduto alla seguente maniera: determinazione delle ampiezze e dei periodi, di ora in ora, per tutte le tempeste microsismiche aventi periodo $T 4$ sec. dell'anno 1949. Le 
misure sono state eseguite sulle registrazioni ottenute con sismografi Galitzin-Wilip per le tre componenti E-W, N-S, $Z$ le cui costanti sono:

\begin{tabular}{|c|c|c|c|c|c|c|}
\hline Componente & $\begin{array}{l}\mathrm{T}_{\mathrm{g}} \\
\text { sec. }\end{array}$ & $\begin{array}{c}T_{P} \\
\text { sec. }\end{array}$ & $k$ & $\mu^{2}$ & $C_{1}$ & $\begin{array}{l}\text { Velocità } \\
\text { di } \\
\text { scorrumento }\end{array}$ \\
\hline $\begin{array}{c}Z \\
N-S \\
F-W\end{array}$ & $\begin{array}{r}10.1 \\
10,2 \\
9.8\end{array}$ & $\begin{array}{l}9,6 \\
9,7 \\
9,45\end{array}$ & $\begin{array}{l}147 \\
139 \\
138\end{array}$ & $\begin{array}{c}-0,003_{8} \\
-0,001_{3} \\
0\end{array}$ & $\begin{array}{l}0.03160_{\mathrm{B}} \\
0.02596_{\mathrm{n}} \\
0.02627_{\mathrm{f}}\end{array}$ & $30 \frac{\mathrm{mm}}{\mathrm{min}}$ \\
\hline
\end{tabular}

Le ampiezze che sono riportate in $\mu$ sui grafici, rappresentano il valore medio delle prime dieci maggiori ampiezze lette nei 10 minuti a cavallo di ciascuna ora. Ciò è stato fatto per ottenere una rappresentazione meno aleatoria e meglio rispondente all'andamento reale di quanto non possa ottenersi col misurare la massima ampiezza nello stesso intervallo come normalmente viene eseguito negli Osservatori. I periodi sono stati misurati con l'approssimazione di 0,1 sec. ma sui grafici sono stati arrotondati a $0,5 \mathrm{sec}$.

Per la comparazione fra agitazione microsismica e situazioni meteorologiche si sono usati tutti i mezzi a disposizione del Servizio Meteorologico dell'Aeronautica Italiana risultati utili e precisamente: a) le carte grandi dell'analisi del tempo elaborato ogni 6 ore (cioè sulle osservazioni delle ore $00,06,12,18$ T.M.G.) dal Centro presso il Ministero dell'Aeronautica; tali carte, sulle quali sono riportati $i$ bollettini delle stazioni facenti servizio internazionale, comprendono tutta l'Europa fin oltre gli Urali, l'Atlantico occidentale e centrale e parte dell'Africa del Nord; $b$ ) le carte delle tendenze barometriche (ciò̀ delle variazioni subite in ciascuna stazione dalla pressione atmosferica nelle ultime tre ore, espresse in millibar e decimi) riguardanti la stessa zona della carta precedente; c) le carte dell'Italia compilate ogni 3 ore a partire dalle 00 T.M.G. con i bollettini (compresa la tendenza barometrica) di tutte le stazioni meteorologiche dipendenti dall'Aeronautica Italiana; $d$ ) tutti i documenti dell'archivio meteorologico, in particolare i registri decadici originali delle Stazioni meteorologiche riportanti per esteso le osservazioni meteorologiche compiute presso ciascuna stazione, e cioè sia le " osservazioni " eseguite ogni 3 ore, sia le " osservazioni ridotte ) eseguite da un buon numero di stazioni ogni ora dall'alba al tramonto.

Per ogni tempesta microsismica si è studiata la situazione meteorologica sulle carte del tempo sopra specificate, consultando, dove occorresse, i registri originali delle stazioni e rielaborando con maggior cognizione di causa (poiché ora ci si può valere dei dati posteriori) quelle analisi che sembrassero non sufficientemente appropriate. 
Spesso per rendere più agevole l'esame dell'evoluzione dei vari elementi si sono costruiti grafici del tipo di quelli riportati nelle pagine seguenti, sui quali sono rappresentati gli andamenti nel tempo di varie grandezze meteorologiche in alcune stazioni (pressione atmosferica, tendenza barometrica, velocità e direzione del vento, stato del mare) e, sulle stesse ordinate, l'andamento dell'ampiezza reale dei microsismi. In questi grafici, mostratisi assai utili e significativi, si riportano i dati di quelle stazioni che la situazione generale indicava come più importanti e decisive, perché poste ad esempio lungo la traiettoria delle perturbazioni. Mediante un esame così minuto e complesso si riesce a farsi un'idea chiara dei rapporti fra situazione meteorologica, vista nella sua unitì e nella molteplicità degli elementi concorrenti, e agitazione microsismica: è allora possibile e legittimo ricercare se esiste un elemento caratteristico della situazione meteorologica cui l'agitazione risulta direttamente collegata.

Questa sarà la prima tappa razionalmente percorsa nell'intento di approfondire le nostre conoscenze sulla natura dei microsismi e sul meccanismo della loro produzione, come anche nell'intento, scientificamente e praticamente non meno importante, di dotare l'indagine meteorologica di un nuovo utile sussidiario. E in questo cammino da percorrere insieme nell'interesse di entrambe le discipline, che sismologia e meteorologia possono collaborare attraverso gli Istituti ed Enti che di esse si occupano.

L'esame delle registrazioni sismografiche del 1949 ha messo in evidenza un certo numero di casi di agitazione microsismica definita con periodi piccoli (generalmente non superiori a $4^{\mathrm{s}}$ ); a volte però l'agitazione di corto periodo compare mista ad altre di periodo più lungo, denunziando una situazione meteorologica complessa ed interessante altre regioni europee od atlantiche, oltre l'Italia, i suoi mari ed il Mediterraneo centro-occidentale. Data la natura della presente ricerca si è ritenuto conveniente tralasciare l'esame approfondito di tali casi di agitazione multipla, poiché meno probativa sarebbe risultata per essi l'individuazione di una causa specifica. Siamo pervenuti così ad isolare le tempeste microsismiche appresso indicate, le quali, è bene sottolineare, rappresentano tutti $\mathrm{i}$ casi individuabili con sufficiente chiarezza di agitazione con periodi non superiori a $4^{\text {s }}$. Di ogni caso si danno brevemente i tratti fondamentali della situazione meteorologica e della sua evoluzione, le caratteristiche morfologicne dell'dgitazione microsismica ed infine $i$ rapporti che risultano fra situazione e microsismi. Alcune tempeste sono invece riportate in detta- 
glio al fine di mostrare in concreto il metodo di lavoro seguito e le ragioni sulle quali hasiamo le conclusioni raggiunte. Tali tempeste particolarmente documentate sono state scelte in modo che risultassero abbastanza ben distribuite fra le varie stagioni e soprattutto fra i vari tipi di tempo caratteristici della regione mediterranea; cosi come è facile verificare, fra esse si trovano rappresentate tutte le traiettorie fondamentali seguite dalle perturbazioni che attraversano il Tirreno. Per ciascuno di questi esempi particolari, oltre ad una descrizione dettagliata della evoluzione del tempo e degli andamenti dei microsismi, si sono riportate alcune cartine ed il grafico di cui si è detto sopra.

Nelle cartine compaiono: a) i fronti mediante il simbolismo internazionale: essi sono rappresentati dalle linee più spesse fornite di semicerchietti (fronti caldi), di cuspidi (fronti freddi) e di semicercljietti e cuspidi alternati (fronti occlusi); la banda nella quale sono i semicerchietti o le cuspidi indicano la regione verso la quale il fronte si sposta; $b$ ) le isoluare, rappresentate dalle linee continue meno spesse e tracciate, come d'uso, di 4 in 4 millibar (nei numeri che le distinguono per lo più sono omesse le cifre delle centinaia e delle migliaia); c) i nuclei delle tendenze barometriche, delimitate dalle linee punteggiate; tali linee sono tracciate per i valori di millibar in millibar, positivi e negativi, come indicato dai numeri accanto e gli spazi racchiusi sono coperti da tratteggio obliquo continuo per i nuclei negativi (pressione in diminuzione) e da tratteggio obliquo discontinuo per i nuclei positivi (pressione in aumento); la densità del tratteggio è proporzionata al valore assoluto delle tendenze.

3. Tempeste microsismiche di origine mediterranea dell'anno 1949. Gennaio 12-13. - Non è un esempio cospicuo come ampiezza di microsismi, ma molto evidente. Durante i giorni precedenti il 13 si è avuto un vastissimo movimento depressionario cloe ha interessato l'Europa da $\mathrm{NW}$ verso il $\mathrm{SE}$, messo in evidenza dalle tendenze barometriche: con moto lentissimo, durato più giorni, un area di tendenze negative di insolite dimensioni apparsa su Inghilterra e Scandinavia si è spostata gradualmente fino a raggiungere il Mediterraneo centrale e le coste Africane. Ciò ha provocato la costituzione e l'approfondimento sul posto di una depressione centrata sull'Italia e attraversata dalla discontinuita tropicale. Durante la griornata del 12 la discontinuità ha dato luogo ad una serie di ondulazioni in moto lento verso E non accompagnata da nuclei di tendenze bene individualoili. Nella giornata del 13 invece allontanatasi la serie delle ondulazioni, in 
concomitanza con l'allontanamento e l'attenuazione della depressione si è verificata una moderata irruzione di masse fredde dal Mediterraneo nord-occidentale verso $\mathrm{SE}$, delimitata da un fronte freddo e accompagnata da un nucleo positivo; il fronte freddo ha attraversato il Tirreno tra le 00 e le 12 circa del 13 gennaio, il nucleo positivo lo ha seguito nella sua parte centrale, a circa 12 ore di distanza. L'attività microsismica benché non notevole, con periodo di $3^{\text {s }}$, si è sviluppata nelle ore pomeridiane del 13: dunque non concomitante alla depressione, allontanatasi già dal giono precedente, e neppure al passaggio del fronte freddo già avvenuto; l'attività concorda con la presenza del nucleo positivo.

Febbraio 2-3. - Si tratta forse della invasione di masse artiche più imponente verificatasi nel corso dell'annata. L'anticiclone atlantico si è trasferito sull'Europa occidentale mentre una depressione piuttosto sensibile staziona sul Mediterraneo con centro sullo Ionio. In queste condizioni nelle ultime ore del giorno 1 si inizia il violento trabocco delle masse artiche dai balcani verso l'Adriatico settentrionale da dove in circa 12 ore dilagano su tutta l'ltalia e sul Tirreno. Infatti dalle ore 12 circa del 2 febbraio a tutto il 3 febbraio si verificano sul Tirreno venti fortissimi nord-orientali e mare grosso o tempestoso. In queste condizioni ci si sarebbe aspettata una agitazione microsismica di piccolo periodo imponente, sia per il vento ed il mare sia per la natura artica e certamente catabatica della massa aerea di invasione sul Tirreno; effettivamente l'agitazione vi è stata ed abbastanza forte ma irregolare e con periodo predominante alto sui 5 sec. Essa si è iniziata nella notte fra l'l ed il 2 con periodo 5 sec ed ha raggiunto la sua massima ampiezza nelle ultime ore del giorno 2; dall'inizio fin verso le ore 08 del 2 è sovrapposta una agitazione di circa 3 sec. Assume perciò particolare significato probativo per il nostro assunto l'esame del comportamento delle tendenze barometriche. Queste tendenze mostrano un forte nucleo positivo che alle ore 18 dell'l compare sulla Venezia Giulia e di li muove verso WSW: alle 00 del 2 è entrato sulla pianura padana con prapagini sul Golfo Ligure e il Tirreno settentrionale; alle 06 il centro è già sulla Liguria occidentale ed a NW della Corsica, ed alle 12 è al largo della Corsica, dopo di che si spinge al largo della Sardegna (dalla parte occidentale) e poi si va attenuando. E chiaro dunque che il nucleo positivo ha interessato solo indirettamente il Tirreno fino alla mattina del 2, mentre ha interessato in pieno il Mediterraneo occidentale. 
E da ricercare dunque in questo comportamento del nucleo positivo la ragione della mancata energia dei microsismi ad origine tirrenica di piccolo periodo prodotti solo dalle zone marginali del nucleo po-

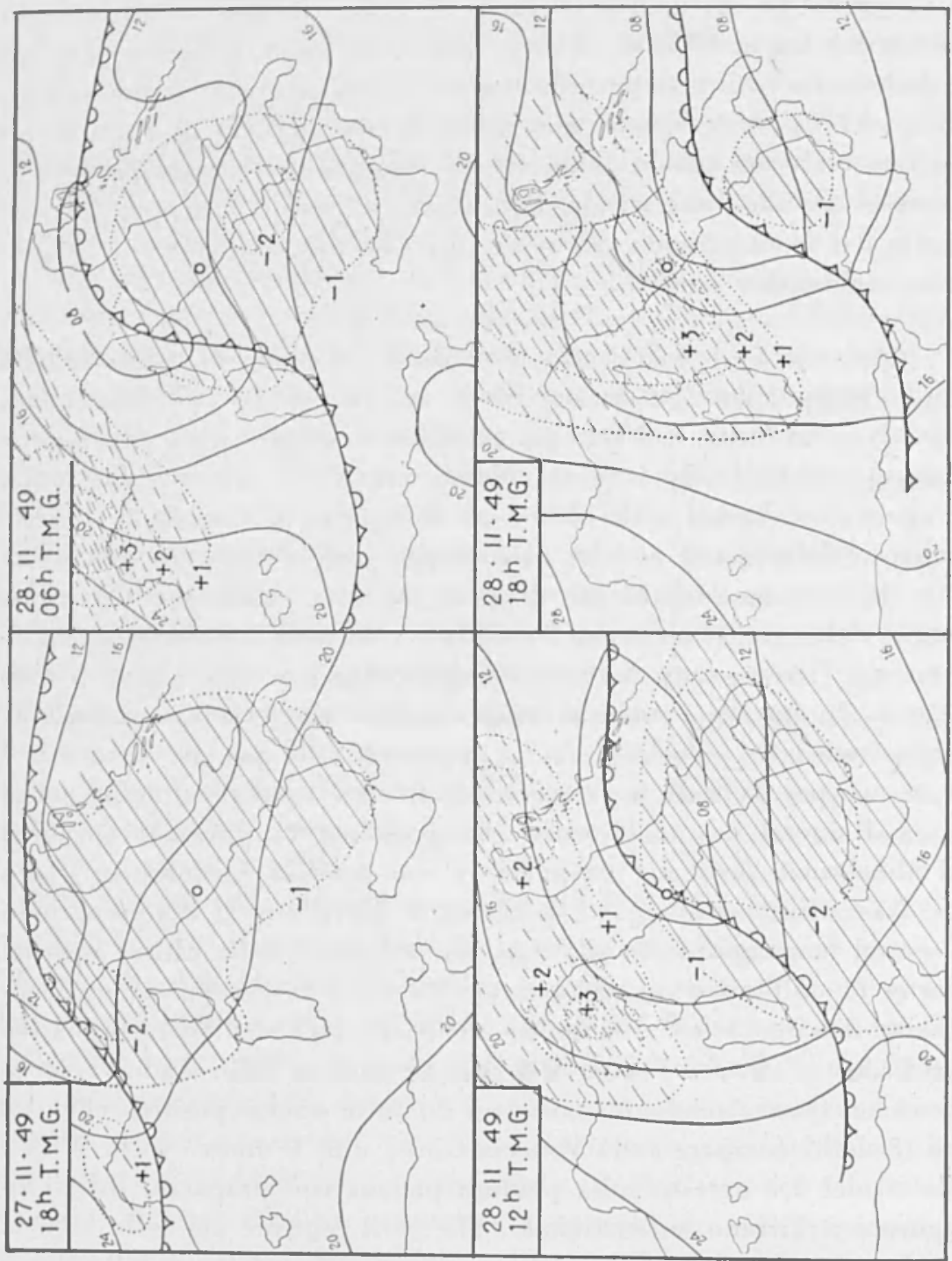

sitivo, e la maggiore energia dei microsismi di $5 \mathrm{sec}$, di origine mediterranea.

Febbraio 27-28 e l Marzo. - Di tale situazione si dà una descrizione dettagliata e documentata. 
Ore 19 del 27. - Una depressione molto profonda interessa tutta l'Europa centrale e settentrionale e si sposta verso $E$ con il centro sul $60^{\circ}$ mentre sull'Atlantico l'anticiclone risulta spostato notevolmente verso $\mathrm{N}$ e tende a spingersi sull'Europa occidentale. Perciò sulla stessa Europa occidentale si hanno forti correnti settentrionali che tendono a dare nuova energia ad una vecchia occlusione che a quest'ora ha cominciato a penetrare dal $\mathrm{N}$ nel Mediterraneo occidentale. Un nucleo positivo che segue detta occlusione comincia ad invadere il Golfo di Lione mentre le tendenze negative molto estese ma non molto profonde interessano tutta l'Italia, il Tirreno e l'Adriatico con centro più profondo sul Golfo ligure. La posizione di quest'ultimo nucleo negativo fa prevedere che l'occlusione piegherà verso $\mathrm{SE}$.

L'agitazione inizia a quest'ora con un periodo di $3,5^{\mathrm{g}}$ ca. cioè piuttosto alto data la modesta ampiezza.

Ore 06 del 28. - L'occlusione ha invaso piuttosto rapidamente il Mediterraneo occidentale, spingendosi con maggiore energia in direzione $\mathrm{SE}$; questo movimento e l'intensità delle correnti settentrionali nel Golfo di Lione hanno provocato il noto fenomeno della

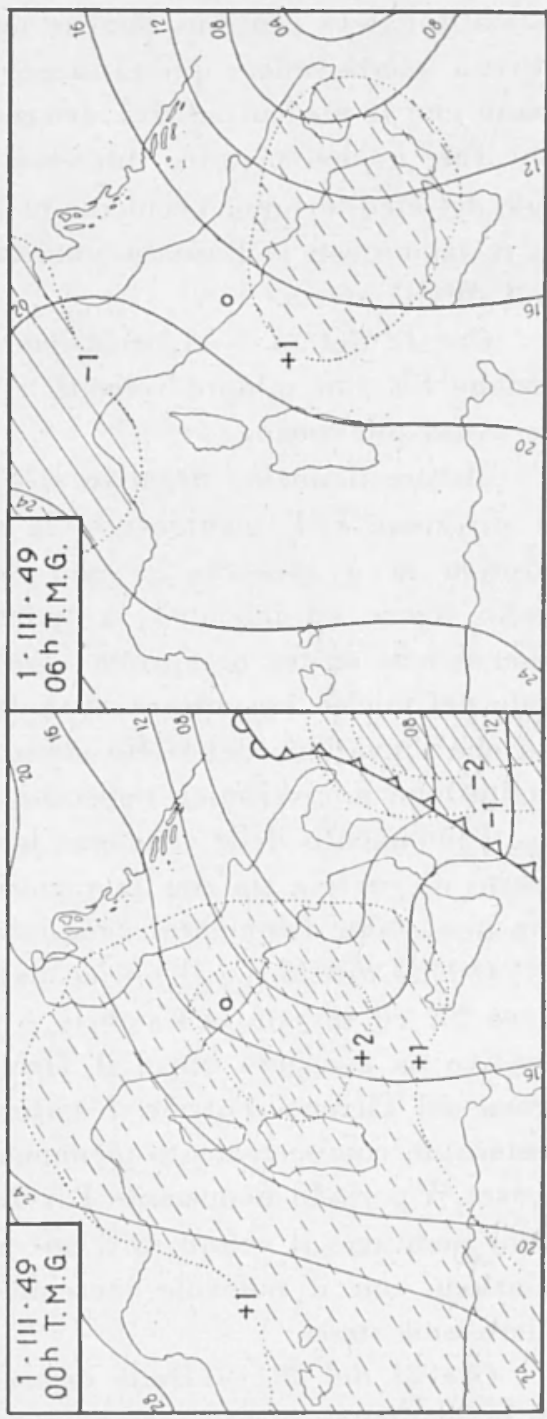
depressione sottovento nel Golfo Ligure; in corrispondenza a tale depressione l'occlusione mostra la tendenza ad assumere la piegatura di ritorno che per solito prelude alla formazione di un settore secondario.

Il nucleo negativo sta attraversando longitudinalmente il Tirre- 
no; ed il suo centro sta avanzando lungo le coste della penisola in direzione $\mathrm{SE}$; anche questa volta il passaggio del nucleo negativo non provoca attività microsismica; anche l'occlusione sul Tirreno settentrionale e centrale, benché notevolmente energica, non produce attività microsismica; questa invece va gradualmente aumentando man mano che il nucleo positivo avanza sul mare aperto.

Tale nucleo peraltro interessa ancora la porzione più settentrionale del Mediterranoe occidentale; il periodo piuttosto lungo di $3,5^{\text {s }} \mathrm{ca}$. in relazione con la limitata ampiezza concorda con la remota origine dell'attività stessa.

Ore 12 del 28. - L'occlusione si è ulteriormente spostata in direzione $\mathrm{SE}$ con minore velocità e la ciclogenesi nell'occlusione non ha avuto più luogo.

Mentre il nucleo negativo si è anch'esso leggermente trasportato in direzione ESE mantenendo la sua notevole estensione, il nucleo positivo si è spostato in una direzione parallela portandosi sul Golfo ligure ed iniziando a spazzare il Tirreno settentrionale. Le osservazioni di tre ore prima (ore 09) mostravano che la zona centrale del nucleo era ancora al di fuori del Golfo Ligure; ciò conferma che l'invasione del Golfo stesso è iniziata successivamente. Infatti l'agitazione microsismica rispecchia in maniera esemplarmente cospicua l'andamento delle tendenze barometriche, sia col suo nuovo aumento di energia sia con la variazione pressoché brusca dal periodo clie nonostante l'aumentata ampiezza si è abbassata a $3^{\mathrm{s}} \mathrm{ca}$.

Ore 18 del 18. - Il nucleo negativo ha continuato la sua marcia verso SE ed investe la Calabria e parte del Tirreno prospiciente; il positivo ha avanzato lungo il Tirreno ed il nocciolo più profondo è ormai sul Tirreno centrale. L'agitazione microsismica continua a presentare un comportamento pienamente fedele all'andamento delle tendenze; il periodo nonostante l'avvicinamento della origine dei microsismi mantiene il valore di 3 sec e non scema; ciò è da mettere in relazione con il notevole incremento che ha subito l'ampiezza dei microsismi stessi.

Ore $21 \mathrm{del} 28$. - Dalle osservazioni di Ponza si può constatare un momentaneo incremento (pur tenendo conto della normale variazione diurna) delle tendenze positive. La gobba del grafico rappresentante l'ampiezza dei microsismi della sola componente NS, che denuncia un lieve incremento, potrelbbe mettersi in relazione con detto aumento.

Ore 00 del 1 marzo. - L'occlusione e le tendenze negative inte- 


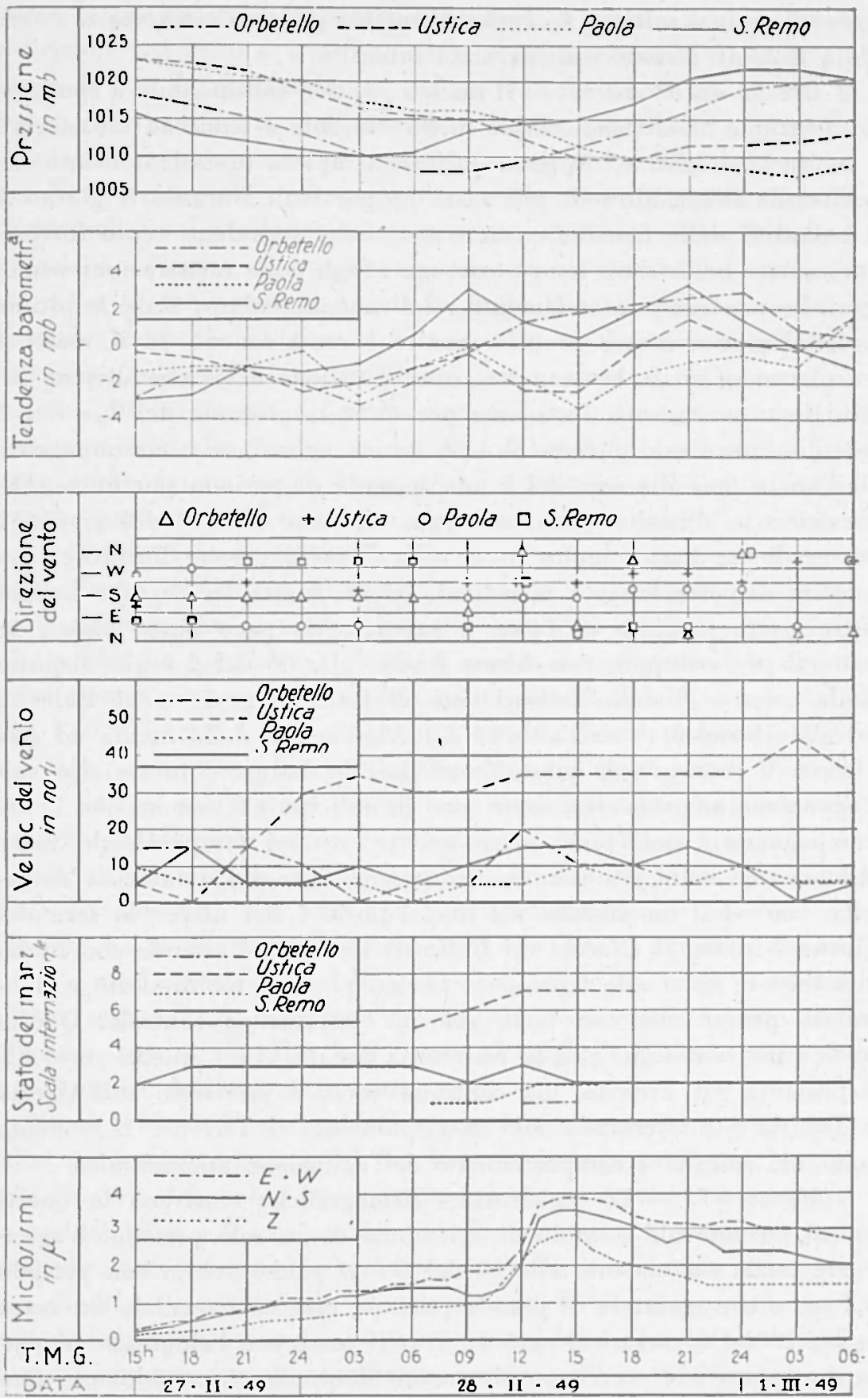


ressano oramai soltanto lo Jonio. Il nucleo positivo viceversa si estende a tutto il Tirreno con invariata intensità .

Ore 06 del $1^{\circ}$ marzo. - Il nucleo positivo continuando a spostarsi in direzione SE diviene sempre meno efficiente e tende ad estinguersi. Marzo 1-2-3-4. - Appena finita la tempesta precedentemente descritta ne inizia altra di più vaste proporzioni. Durante il giorno 1 i bollettini delle Stazioni costiere tirreniche segnalano vento fortissimo e mare addirittura tempestoso, ma i fogli delle registrazioni sismografiche mostrano un andamento relativamente calmo; verso le ultime ore del giorno invece proprio mentre $i$ venti cadono ed il mare si va placando, inizia bruscamente una agitazione di $5^{\mathrm{s}}$ che diviene subito forte e continua fortissima per tutta la giornata del 2 e del 3 per diminuire solo durante il 4 . A questa agitazione è accompagnata dall'inizio fino alla sera del 2 una seconda di periodo più breve. Osservando la situazione meteorologica, troviamo alle 18 del giorno 1 un gradiente barico molto forte sulla Francia e zone limitrofe, con isobare disposte lungo $\mathbf{i}$ meridiani, ed un fronte freddo che ha già attraversato il Golfo di Lione e superato da poco l'arco alpino in tutto il suo sviluppo; tale fronte freddo alle 06 del 2 è già disposto dalla zona a $S$ delle Baleari fino all'Italia centrale e ai Balcani, ed alle 18 del 2 va dall'interno dell'Algeria a S della Sicilia ed alla Grecia. Il passaggio di questo fronte freddo dunque è in anticipo sull'agitazione microsismica, come sono in anticipo i fenomeni che lo accompagnano (venti forti, mare agitato, rovesci temporaleschi ecc.). Ancora una volta risultano contemporanei invece l'andamento dell'agitazione ed il movimento dei nuclei positivi nel mare: la sera del giorno 1 infatti si affaccia sul Golfo di Lione, e si estende con intensità minore sul Golfo ligure, un nucleo positivo molto netto e delimitato proveniente con forte velocità dall'Europa centrale. Questo nucleo poi non segue più la traiettoria usuale, che è quella verso SE e passante sul Tirreno, ma continua verso $S$ puntando sull'Algeria e Tunisia e interessando solo marginalmente il Tirreno. E evidente come ciò spieghi il comportamento dell'agitazione microsismica.

Marzo 6-7. - Le registrazioni sismografiche mostrano in questi giorni un notevole esempio di agitazione di un solo periodo. L'agitazione inizia esattamente alle 00 del 6 e si palesa subito con periodo 3,5 sec e con carattere di grande purezza, essa va crescendo fin verso le ore $12 \mathrm{del} 6$, mentre il periodo di pari passo con l'ampiezza aumenta e si porta a 4 sec; successivamente diminuisce gradualmente fino a divenire debole con periodo 3 sec nella sera del 7. La situazione 
meteorologica generale del giorno precedente 5 marzo, è la seguente: l'anticiclone atlantico si è ritirato nella sua sede normale e poiché ha ripreso le sue caratteristiche di centro d'azione anche in quota, le perturbazioni originate nell'Atlantico Nord-occidentale, a S della Groenlandia descrivono un imponente arco di cerchio investendo l'Europa Nord-occidentale e proseguendo in direzione dell'Italia. Una di tali perturbazioni è alle 00 del 6 centrata sul Tirreno, ove il settore tropicale è collegato ad una depressione che prodottasi sul Golfo ligure circa 12 ore prima si ̀̀ andata gradualmente approfondendo e spostando verso SE. $\grave{E}$ necessario notare che se l'agitazione microsismica fosse collegata al centro depressionario, non avrebbe avuto situazione più favorevole di questa per iniziare prima e mostrarsi a quest'ora e cioè alle 00 del 6 , in pieno sviluppo. Ancora più anticipato avrebbe dovuto essere il suo inizio se essa fosse collegata, come potrebbe anche ragionevolmente supporsi, con $i$ nuclei di tendenze negative: infatti un forte nucleo negativo ha attraversato in tutta la sua lunghezza il Tirreno ed ora trovasi nella sua parte meridionale. Il collegamento con il nucleo negativo potrebbe sembrare a priori molto probabile, perché è appunto il nucleo negativo che prepara il cattivo tempo, ciò̀ apre la marcia delle perturbazioni frontali e ne segna l'attività, come è giustificato da considerazioni teoriche e provato dalla esperienza. Invece l'agitazione microsismica per iniziare attende l'arrivo sul Tirreno del nucleo di tendenze positive; tale nucleo precedentemente debole e proveniente da NW, si rinforza fra le osservazioni meteorologiche delle 21 , che lo segnalano appunto debole e circoscritto alla Sardegna nord-occidentale, e le osservazioni delle 00 , che lo danno notevolmente più efficiente e situato sulle coste sarde nord-orientali ed al largo di esse. Successivamente il nucleo positivo, seguendo la traiettoria del negativo, attraversa nel suo asse il Tirreno, mostrando alle 12 il centro al largo di Ponza e raggiun. gendo le coste siciliane nella notte.

Marzo 9.10. - Una agitazione di moderata intensità emerge gradualmente intorno alle ore 07 del 9 corr. periodo sui $3 \mathrm{sec}$, cresce fin le 18 circa del giorno stesso mostrandosi non forte ma assai pura con periodo di 3 e 5 sec, poi decresce lentamente. La situazione meteorologica presenta una perturbazione sul Tirreno dovuta alla presenza di un settore tropicale proveniente da W. Il campo delle tendenze barometriche, benché piuttosto sovrabbondante di particolari che non è sempre agevole coordinare, mostra nelle ore dell'agitazione microsismica certamente una distribuzione positiva sul Tirreno, indi* 
ce indublio della presenza di un nucleo la cui traiettoria rimane tuttavia non bene determinabile.

Marzo 19, ore 00 . - La precedente situazione barica sull'Europa

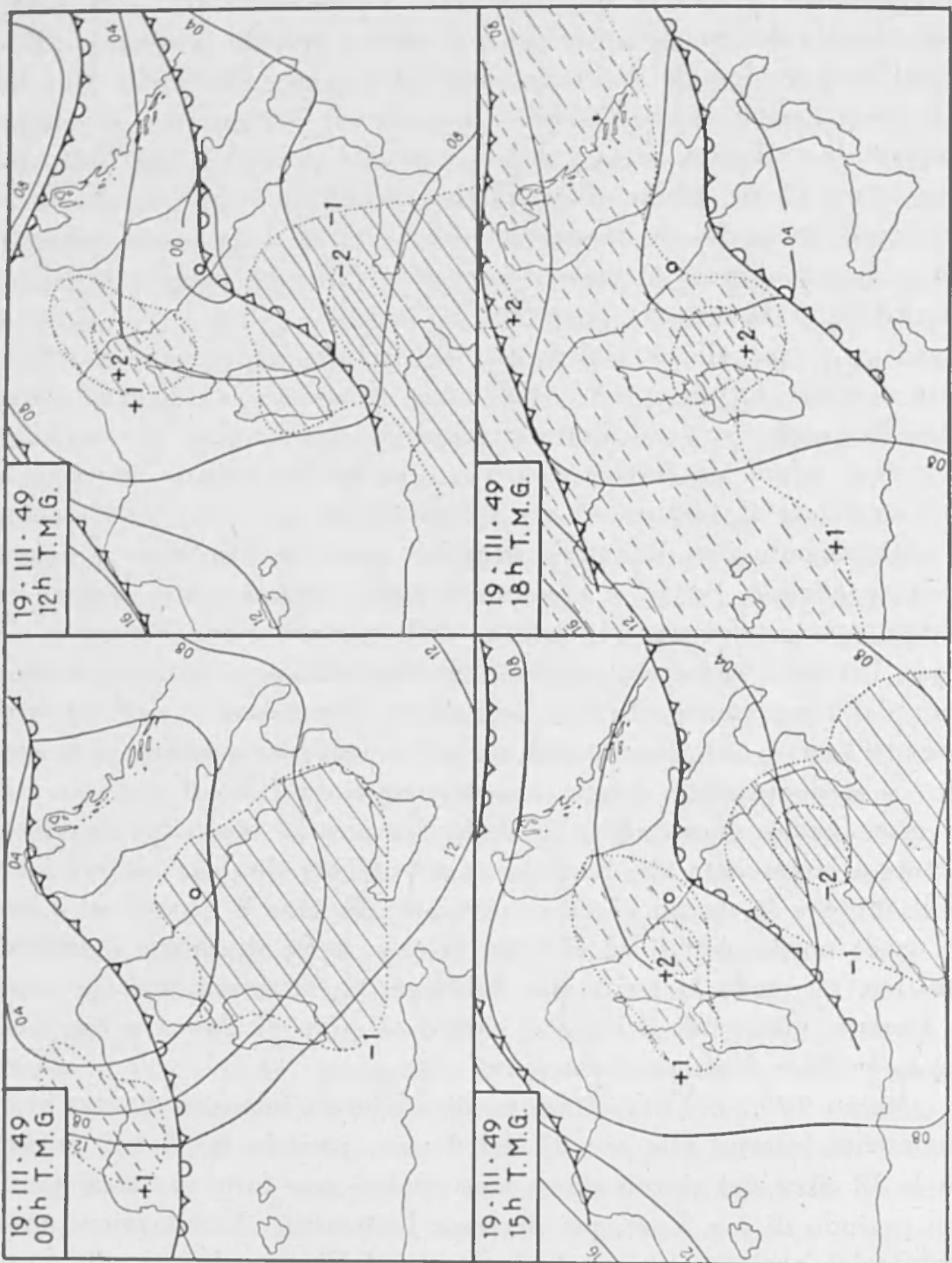

occidentale e sull'Atlantico, con isohare ravvicinate correnti da $\mathrm{N}$ a $\mathrm{S}$, ha provocato una depressione sul Golfo ligure che ora si è allargata ed inizia il suo movimento verso SE. Ad essa è collegata un'occlusione a carattere freddo proveniente dal $\mathrm{N}$ ed ora 
tendente ad assumere anch'essa una direttrice di marcia verso SE. In accordo con tale situazione la carta delle tendenze mostra un nucleo negativo non profondo sul Tirreno centrale e meridionale ed adiacenze, ed un nucleo positivo che provenendo dal golfo di Lione sta per fare il suo ingresso nei mari italiani passando a nord della Corsica. L'agitazione microsismica va gradualmente prendendo consistenza e mostra un periodo di 4,5-5 sec, il periodo cioè che compete a tale distanza da Roma delle cause agenti.

Ore 12. - L'occlusione fredda ed il minimo ad essa collegato (che ne provoca un'ondulazione senza peraltro aver energia sufficiente per trasformare l'ondulazione in un vero sistema caldofreddo con processo secondario di occlusione) si sono trasferiti lungro il Tirreno ed ora ne occupano la parte centrale. L'agitazione microsismica, se fosse originata nella

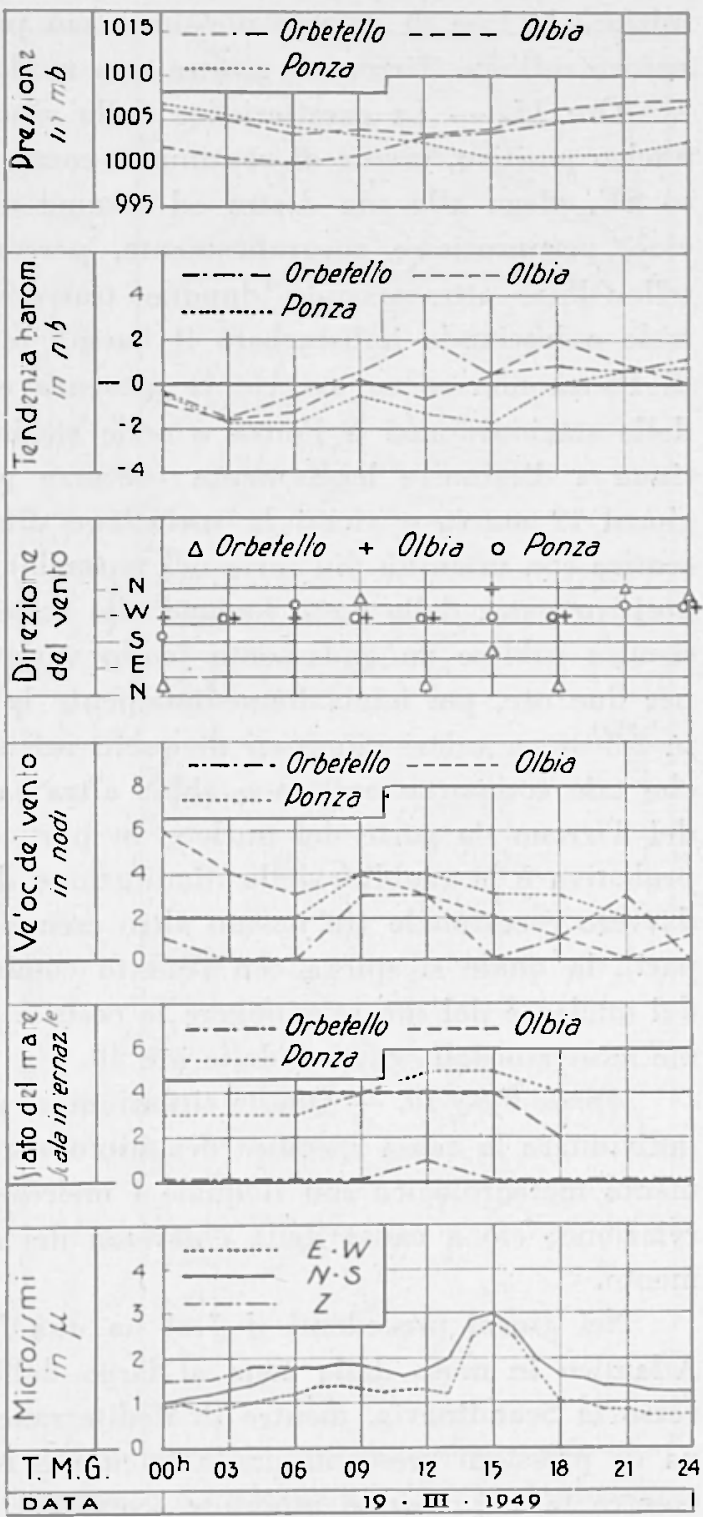
zona centrale della depressione o lungo la linea del fronte, dovrebbe attingere ora la massima ampiezza ed il minimo periodo, dato che la depressione è pro- 
prio centrata su Roma ell anzi si è ulteriormente approfondita nelle ultime ore. L'agitazione invece pur continuando a crescere d'intensità e malgrado ciò accennando a diminuire di periodo, deve ancora iniziare la fase di attività massima; ciò perché il nucleo positivo è ancora sull'alto Tirreno e mostra una moderata intensità.

Ore 15. - La caratteristica della situazione in esame è che il nucleo positivo, invece di continuare come sempre la sua marcia verso $\mathrm{SE}$, piega alla sua destra ed assume una direttrice verso WSW cioè, per precisare geograficamente, percorrere la traiettoria Orbetello-Olhia, attraversando dunque trasversalmente il Tirreno centrale e lasciando indisturbato il bacino meridionale. Già all'altezza di Ponza non se ne avverte la presenza come mostrato dal grafico delle stazioni; anzi a Ponza e nelle stazioni a $S$ la pressione continua a diminuire leggermente (occorre peraltro avvertire che nei giorni 19 marzo e vicini la variazione diurna della pressione si fa sentire con intensità più forte del normale). Proprio mentre il nucleo positivo passa dalle coste toscane alle coste sarde l'agitazione microsismica subisce un andamento molto variato: aumenta bruscamente per due ore, poi inizia immediatamente la diminuzione per tornare in $2-3$ ore a valori più bassi di quelli iniziali. Sembra effettivamente che tale comportamento non ablia altra causa che l'attraversamento del Tirreno da parte del nucleo; in particolare ciò che sembra più probativa è la rapidità della diminuzione dell'intensità microsismica, davvero eccezionale (in nessun altro esempio se ne è riscontrata una pari), la quale si spiega con l'effetto combinato dell'allontanamento del nucleo e del suo raggiungere la costa e quindi la terra ferma, come mostrato dalla cartina delle ore 18.

Aprile 7-8-9-10. - Questa situazione si presta particolarmente per individuare la causa specifica dei microsismi, o più esattamente l'elemento meteorologico con il quale $\mathrm{i}$ microsismi sono direttamente in relazione; ciò a causa della chiarezza dei fenomeni nel loro svolgimento.

Nei giorni precedenti il 7 si ha una forte depressione nell'alto Atlantico in moto dalla zona al largo dell'Inghilterra settentrionale verso la Scandinavia, mentre il Mediterraneo è in un'amplissima zona di pressioni crescenti gradualmente e regolarmente. Il giorno 6 , mentre la depressione atlantica scompare verso $\mathbf{N}$ ed il forte nucleo di tendenze positive entra in terra scandinava nel momento stesso in cui l'agitazione microsismica di lungo periodo rilevata a Roma si attenua e cessa rapidamente, si delinea la rigenerazione di una 
occlusione di ritorno tropicale sull'Inghilterra meridionale e la Francia. Poiché contemporaneamente la pressione sul Mediterraneo occidentale arresta il suo andamento di lenta ascesa e diviene sede di

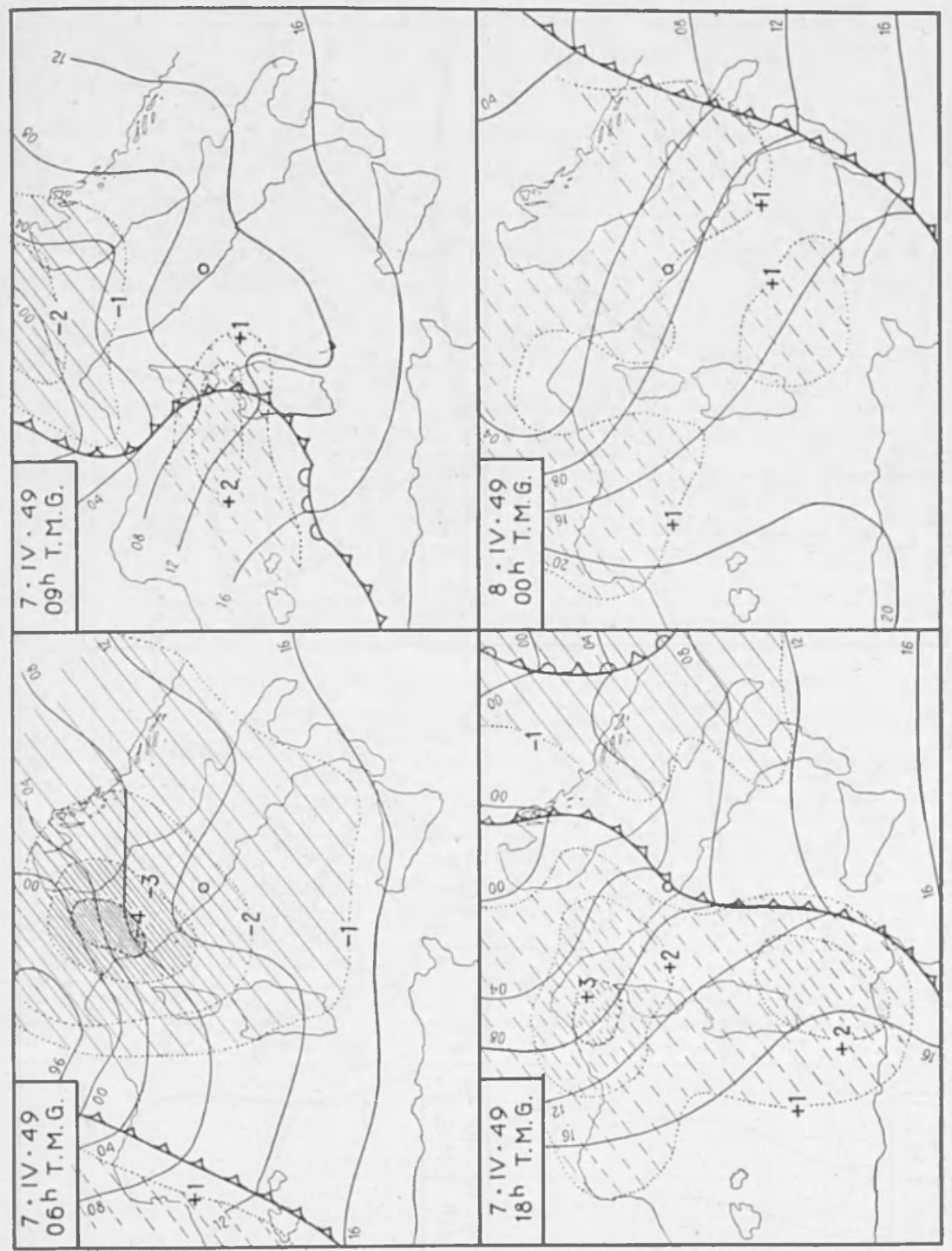

grossi nuclei di tendenze negative è da presumere che la depressione collegata all'ansa dell'occlusione sia in rapido approfondimento ed in veloce moto verso $\mathrm{SE}$ e che l'occlusione si trasformi in settore intermedio. 
Infatti la situazione alle ore 06 del 7 , per quanto riguarda il Mediterraneo, è caratterizzata da due fatti specifici: l'area di tendenze negative assai forti si è spostata verso $\mathrm{E}$ interessando tutta l'Italia

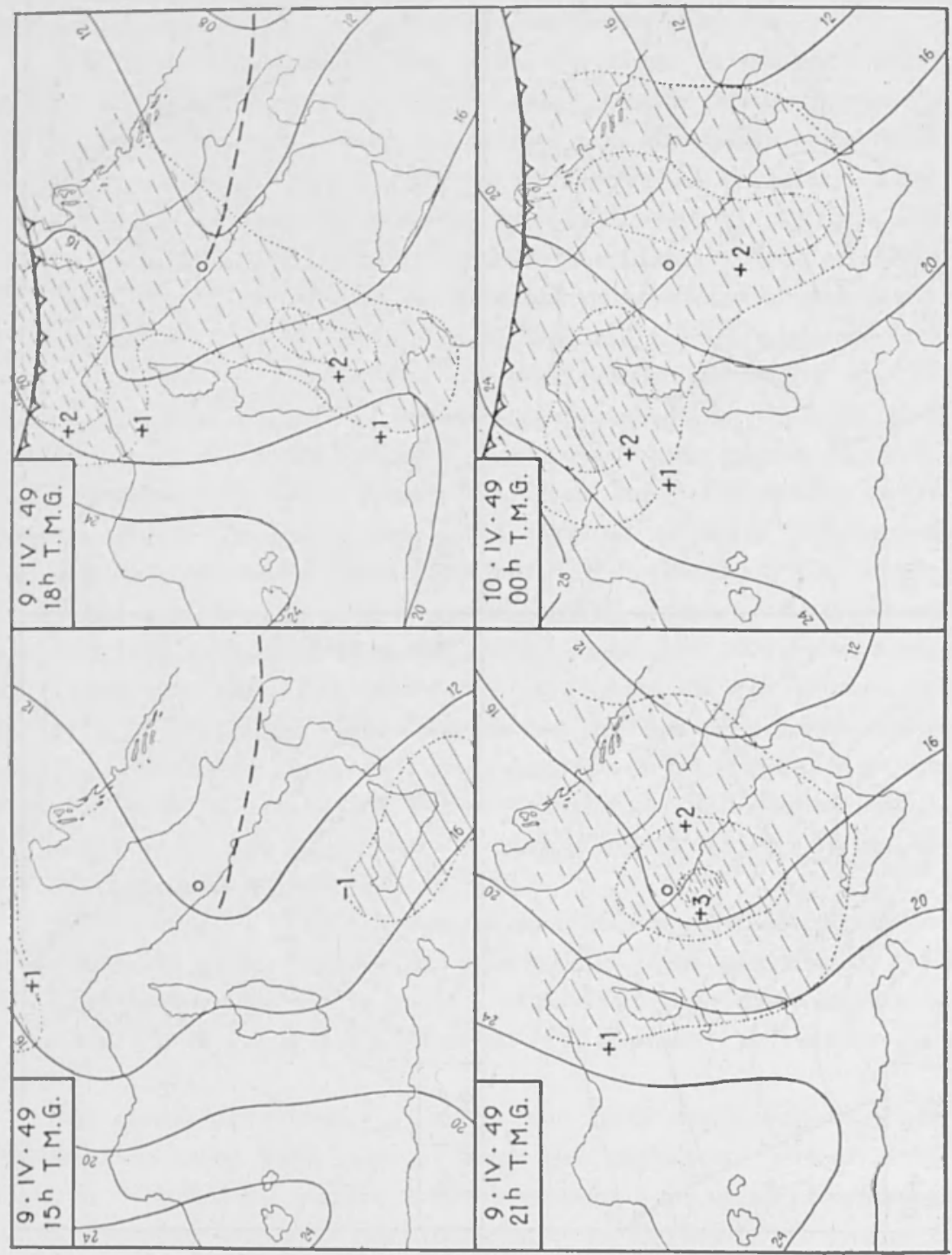

e le adiacenze, ed un fronte freddo ha fatto irruzione da W e NW lungo tutta la costa ispano-francese fino al golfo di Lione. A questo punto è di basilare importanza notare che l'investimento brusco dei 


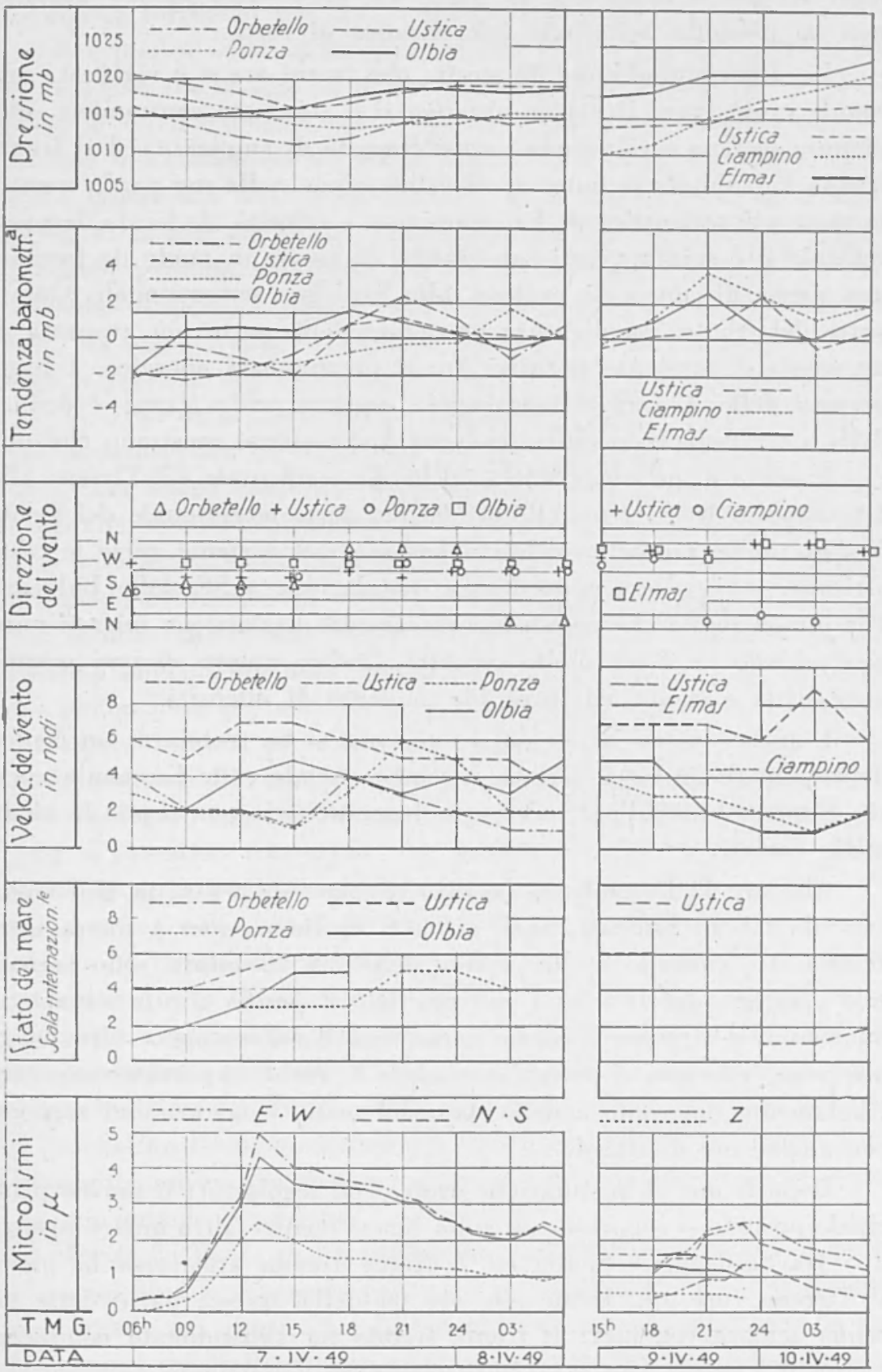


mari italiani e dell'Ttalia da parte del grossissimo nucleo negativo non ha prodotto agitazione microsismica di sorta.

La situazione alle ore 09 mostra che in tre ore si è verificata una rapida evoluzione: il nucleo negativo si è attenuato centrandosi sulla pianura padana e riducendosi sensibilmente di ampiezza, ed il fronte freddo ha subito una notevole diversificazione nelle sue parti: mentre la parte più settentrionale ha camminato a velocità moderata, la parte centrale si è spinta avanti con grande decisione in modo da formare una specie di cuneo già proteso sulla Sardegna settentrionale. Questa parte del fronte è addirittura preceduta dalla parte più avanzata di un nuceo di tendenze positive che si preannunzia energico. I barogrammi delle stazioni meteorologiche costiere sarde (come si deduce dalla " caratteristica " della tendenza barometrica) mostrano che una rapida salita di pressione inizia sul bordo occidentale del Tirreno alla latitudine di Roma circa alle ore 08 . La parte meridionale del fronte freddo, infine, pur muovendosi abbastanza velocemente verso le coste africane, subisce una momentanea ondulazione a SE delle Balneari. E a questo punto che comincia bruscamente l'agitazione microsismica con periodo di 3 sec molto regolare (indice questo di una origine circoscritta e vicina) ed in rapido aumento di intensità.

È anche qui essenziale notare che non si ha traccia di un centro depressionario in tutta la zona, il centro essendo sulla Germania: perciò di microsismi collegati al centro depressionario non si può in alcun modo parlare.

Alle ore 12 le tendenze positive si sono estese e tutto il Tirreno centrale (il meridionale, anzi, già a $\mathrm{S}$ di Roma, non è finora interessato alla vicenda): sulla cartina delle ore 12 infatti sono segnati solo i valori superiori ad $1 \mathrm{mb}$ positivo. É perciò che la situazione microsismica raggiunge proprio a quest'ora il suo massimo: altra causa più immediata non si potrebbe pensare. $\mathrm{E}$ anche opportuno osservare l'andamento del vento e dello stato del mare, $\mathrm{i}$ cui massimi seguono con alcune ore di ritardo.

Dopo le ore 12 la situazione evolve con regolarità: il nucleo principale positivo si è portato sul golfo ligure mentre altro nucleo avanza a S fra Sardegna e Sicilia ed il fronte freddo attraversa in pieno il Tirreno (ore 19). Infine alle ore 00 dell' 8 questa parte della vicenda sembra conclusa: il fronte freddo ha abbandonato completamente il Tirreno, che è in vastissima zona debolmente positiva. Occorre notare che mentre l'agitazione si riduce considerevolmente, ed 
ha solo una ripresa verso le ore 12 del giorno 8 , il vento ed il mare rimangono fortissimi per tutti i giorni 8,9 e 10 .

Nel quadro della situazione descritta si inserisce un episodio per noi notevole fra la sera de giorno 9 e le prime ore del 10. Se osserviamo le situazioni dalle ore 16 del 9 in poi non troviamo nulla di notevole nel campo barico ed in quello frontologico: isobare abbastanza lasche con andamento da NW a SE sul Tirreno, in accordo con la direzione dei venti, invero però ancora più forti di quanto lascerebbe presumere il gradiente barico. Il centro di previsioni dell'Aeronautica però, in concordanza con una debole e ristretta saccatura da E, ha segnato una "linea di convergenza ) (la tratteggiata nella figura a pag. 192) che attraversa l'Italia centrale e termina nel 'Tirreno, poco al largo delle coste laziali. Mentre i fenomeni meteorologici diretti poco provano sul'esistenza e consistenza di tale linea di convergenza, una buona conferma viene dall'agitazione microsismica. Si ha infatti che mentre l'agitazione già segnalata nei giorni precedenti continua ad attenuarsi, si rende visibile fra le ore 18 e 19 una agitazione di periodo brevissimo di circa 2 sec, la quale raggiunge il suo massimo intorno alle 00 del 10 , per poi decrescere e svanire. Ora se esaminiamo landamento delle tendenze barometriche troviamo un fatto che, se non fosse segnalato dalle osservazioni, difficilmente sarebbe potuto essere intuito: un nucleo molto ben definito di tendenze positive, inesistente alle ore 15 (solo una zona debolmente positiva a sud della Sardegna) procede da sud-ovest rinforzandosi notevolmente durante il cammino trasversale alle isobare, fino ad investire il mare fra Ponza e Roma intorno alle 21. $\grave{E}$ di grande interesse notare che esso provoca nella zona un indebolimento notevole del vento, anzi alle ore 21 Ponza segnala un vento non forte da sud-est, ciò̀ proprio opposto a quello dominante su tutto il Tirreno (dato controllato direttamente); poco dopo, finito il fenomeno, Ponza torna ad allinearsi con 'landamento generale. Dunque in quelle ore "qualcosa è avvenuto ) fra Roma e Ponza, senza fenomeni vistosi, anzi con soltanto un indebolimento momentaneo del vento; ma si è avuto l'arrivo di un circoscritto nucleo positivo, e l'agitazione di corto periodo l'ha segnalato con grande precisione.

Aprile 25-26-27. - La situazione meteorologica, rimasta a lungo calma sul Mediterraneo dopo la precedente tempesta va gradualmente peggiorando nei giorni 23 e 24 culminando con la formazione di una depressione sul Mediterraneo occidentale e con la comparsa nella zona di un insolito numero di fronti tropicali ed intermedi, alcuni prove- 
nienti da NW altri formatisi o rigeneratisi sul posto. Tutto l'insieme accompagnato da forti piogge e venti dai settori occidentali, muove successivamente verso $\mathrm{E}$ giungendo fra il 24 e il 25 sul Tirreno e sull'Italia. L'agitazione microsismica tuttavia non inizia che nel tardo pomeriggio del 25, con periodo $3-3,5 \mathrm{sec}$ e con aspetto alquanto complesso; essa raggiungre la massima intensità intorno alle 00 del 26, poi è sommersa da un'altra agritazione di periodo più lungo (6 sec) che iniziata più tardi finisce per divenire predominante. Pur con la complessità della situazione meteorologica, risulta evidente che l'agitazione di breve periodo inizia solo quando le tendenze positive piuttosto incerte finché erano situate sul Mediterraneo occidentale compaiono e si consolidano sul Tirreno centrale, cosa che avviene appunto nella seconda metà del giorno 25. Successivamente tutto si allontana verso $\mathrm{SE}$ mentre l'agitazione come si è detto è soverchiata da altra di periodo di 6 sec. A proposito di quest'ultima componente, abbiamo notato che le carte delle tendenze barometriche mostrano nella giornata del 26 l'arrivo di un potente nucleo positivo sul golfo di Guascogna, il cui collegamento con l'agitazione microsismica balza evidente; tuttavia non ci soffermiamo qui su questa estensione della ricerca presente, ripromettendoci di tornarci in un secondo tempo.

Maggio 1-2-3. - Si ripresenta in questo periodo, con caratteri aggravati dalla maggiore energia in giuoco una situazione analoga a quella precedentemente segnalata nella terza decade. di aprile: una vasta zona depressionaria centrata sul Mediterraneo occidentale ed interessante l'Italia con tempo cattivo ovunque per la presenza e l'attività di numerose perturbazioni. A tutto ciò si accompagna una vistosissima agitazione microsimica nella quale i periodi prevalenti fra tutta una gamma presente, sono quelli di $5-5,5$ sec e di 4 sec. Anche in questo caso è visibile l'accordo fra l'agitazione di 4 sec comparsa intorno alle 19 del giorno 1 e l'arrivo sul Tirreno alla stessa ora del primo nucleo positivo dopo già quasi 24 ore di tempo molto perturbato. Tuttavia la molteplicità delle perturbazioni ed il loro rapido succedersi rendono piuttosto problematica la individuazione dei comportamenti dei singoli elementi; in altre parole la situazione sia meteorologica che microsismica è talmente perturbata che l'indicazione di una causa invece di un'altra diviene poco convincente. A noi basta notare che questo caso non contraddice al nostro assunto, anche se è troppo confuso per costituirne una prova.

Maggio 13. - Questa è la situazione di tutto il 1949 nella quale il collegamento tra agitazione microsismica e situazione meteorologica 
appare più circoscritta nello spazio e quindi più difficoltosa nella ricerca delle cause dirette. Le carte generali del tempo non mostrano altro che la presenza di una goccia di aria artica residua il cui limite meridionale va dalla penisola del Gargano alla Sardegna; la pressione è molto livellata, il mare è calmo e vi è assenza di venti generali ma si verificano forti temporali locali nell'interno della goccia artica, cioè sull'Italia settentrionale e centrale; così forti temporali si verificano sul Lazio tra le ore 12 e le 18 . Le carte delle tendenze barometriche non mostrano la presenza di nuclei definiti positivi o negativi, a meno che questi nuclei siano talmente localizzati da interessare praticamente una sola stazione o da sfuggire alle osservazioni. L'agitazione microsismica pur rimanendo sempre moderata, mostra due episodi distinti: l'uno nelle prime ore del giorno tra le ore 01 e 06 , con periodo di 2,5-3 sec, e l'altro nel pomeriggio tra le ore 12,30 e le 19 circa, con periodo brevissimo di appena $1-1,5$ sec e con forte componente verticale. Questa seconda agitazione non può essere collegata altro che ai temporali già segnalati verificatesi sul Lazio e sul mare adiacente: quanto alla causa specifica peraltro non si posono fare ovviamente, con i dati a disposizione, altro che ipotesi più o meno convincenti. Sarebbe necessaria infatti una documentazione, per così dire micrometeorologica che nessun servizio finora possiede; per casi analoghi sarà utile del resto, come messo in luce da numerosi Autori, una rete di microbarografi registratori che è in corso di realizzazione da parte dell'Istituto Nazionale di Geofisica in collaborazione con il Servizio Meteorologico dell'Aereonautica. Le registrazioni barografiche di Roma mostrano ad ogni modo un andamento pulsante della pressione nelle ore del temporale. Quanto alla spiegazione della prima agitazione, cioè quella mattutina di periodo maggiore siamo in grado di trarre dai dati un solo elemento sicuro: esattamente in quelle ore e non in altre si sono avuti temporali sull'alto Tirreno segnalati in particolare dalla Stazione meteorologica dell'isola di Pianosa.

Maggio 22-23. - L'agitazione microsismica di breve periodo (3-3,5 sec) emessa nei giorni 22 e 23 trova corrispondenza nella situazione perturbata sul basso Tirreno e sullo Ionio, ove insiste un nucleo di tendenze positive invero non molto definito e conseguente al passaggio avvenuto il giorno innanzi di un fronte freddo. La situazione nel complesso è poco significativa.

Giugno 24-25. - Guidata dalle correnti in quota, aventi una direttrice da $\mathrm{SW}$ una depressione barica collegata al settore tropicale formatosi sull'Algeria si sposta verso la Sardegna ed il Tirreno che 
raggiunge nelle prime ore del giorno 24 . La perturbazione è preceduta da un nucleo negativo che interessa l'Italia ed il Tirreno fin dal pomerigrio del 23 ed è seguita da un nucleo positivo che entra nel Tirreno centrale proveniente dalla Sardegna nella tarda mattinata del 24. L'agitazione microsismica emerge gradualmente appunto dopo le ore 10 del 24 con periodo di quasi 3 sec e raggiunge la massima intensità (per altro moderata) ed una notevole purezza intorno alle 06 del 25; in questa ora il nucleo che si è spostato molto lentamente, è centrato sul Tirreno centrale. Successivamente il nucleo entra nella terra ferma spostandosi verso $\mathrm{E}$ e l'agitazione decresce e scompare.

Luglio 7-8. - Dopo le ore 22 del 7 compare una agitazione di circa 3 sec che rimane sempre assai debole per scomparire circa 24 ore dopo. Data la lieve entità dell'agitazione il caso non merita un esame dettagliato; comunque la situazione meteorologica è caratterizzata da un settore tropicale che dal Mediterraneo occidentale si porta sul Tirreno e l'Italia mostrando un fronte freddo più attivo del caldo seguito da un distinto nucleo positivo che sulla carta delle tendenze delle ore 00 è segnato sul Tirreno centro-settentrionale. L'inizio dell'agitazione dunque coincide con la comparsa del nucleo. Successivamente peraltro la situazione isallobarica diviene meno chiara con l'indebolirsi degli altri elementi della perturbazione.

Luglio 19-20. - Altra tempesta microsismica molto debole, che è riportata solo per completezza, è quella manifestatasi nei giorni 19 e 20 luglio con la presenza indistinta di molti periodi fra 1 e 3 sec cui si sovrappone successivamente altra agitazione con periodi più lunghi ma anch'essi variabili fra i 4 e i 5 sec. Le carte della situazione mostrano alle ore 00 del 19 un fronte freddo sull'Italia settentrionale ed il Golfo ligure in cammino verso sud: è pure evidente, benché non segnata, una discontinuità sul Mediterraneo occidentale ove dalle ore 12 cominciano venti settentrionali che poi si estendono all'Italia. Contemporanea alla diffusione dei venti è la diffusione delle tendenze positive; la complessita della situazione spiega la molteplicità dei periodi presenti. Questo esempio benché nel complesso di debole entità, può essere interessante per la pluralità di cause piuttosto vicine ma complessivamente non è molto pertinente alla ricerca attuale.

Agosto 3-4. - Elemento caratteristico della situazione meteorologica è una distribuzione barica stagionale che si estende in quota fino alla superficie isobarica di 500 billibar, perciò le correnti aeree e le perturbazioni frontali provenienti dall'Atlantico peraltro poco intense procedono verso $\mathrm{E}$ interessando oltre l'Europa centrale solo l'Italia 
settentrionale. Collegati con una di tali perturbazioni una coppia di nuclei di tendenze (negativo e positivo) attraversa il golfo Ligure e l'alto Tirreno senza interessare in nessun modo le zone più a sud. La valutazione delle tendenze negative con quelle positive avviene nella predetta zona intorno alle ore 03 del giorno 3 , ed il nucleo positivo più intenso vi passa nella notte fra il 3 ed il 4 . La tempesta microsismica non si manifesta molto intensa, ma è assai nitida e conferma le relazioni già stabilite fra microsismi e vicende meteorologiche; essa inizia gradualmente nelle prime ore del 3 con periodo di $3,5 \mathrm{sec}$, soggiunge la massima intensità con periodo leggermente accresciuta circa 24 ore dopo, poi si attenua.

Agosto 18-19. - Spesse volte avviene che le perturbazioni presenti siano più d'una: in tali casi generalmente l'agitazione microsismica diviene molto ampia e confusa, in modo tale che non si può condurre una ragionevole indagine sulle relazioni tra essa e le presumibili cause: tutti gli elementi sono praticamente presenti, per cui alla complessità della struttura dei microsismi fa riscontro, globalmente, la complessità della situazione meteorologica. La situazione del 18-19 agosto ha una particolarissima importanza perché permette l'analisi dell'agitazione in due componenti, in accordo davvero sorprendente con la chiara duplicità delle perturbazioni in viaggio sul Tirreno. Nel grafico dell'ampiezza dei microsismi sono stati riportati gli andamenti dei due tipi di agitazione, perfettamente identificabili e seguibili nel corso di tutta la tempesta. Sareble agevole considerare, come abbiamo fatto nelle altre situazioni, gli andamenti dei periodi in rapporto alla distanza dei nuclei di tendenze positive, per constatarne ancora una volta il legame; ma poiché ciò già è da considerare provato mentre l'interesse specifico della situazione in esame è alquanto diverso, prescinderemo qui dai periodi, limitandoci a segnalare la loro brevità per ambedue i tipi (nessuno dei due raggiunge mai i $3,5 \mathrm{sec}$ ).

Ore 12 del 18. - La situazione meteorologica è comandata dall'anticiclone atlantico che spinge un promontorio sull'Europa occidentale; di conseguenza le linee di corrente da NE verso SW. Sul Mediterraneo una depressione precedentemente formatasi ha provocato l'ondulazione del fronte tropicale che ora è in processo di occlusione. I venti in prevalenza nord-occidentali sono mediocremente forti.

Poco più a nord del fronte tropicale vi è un fronte freddo proveniente dai Balcani ed in moto verso $S W$ che ancora non interessa il Tirreno. I nuclei delle tendenze barometriche hanno una disposizione tipica rispetto ai fronti: un nucleo negativo sul Tirreno meri- 
dionale, un nucleo positivo non molto esteso sul Tirreno centro-occidentale dietro il fronte freddo tropicale ed un secondo nucleo positivo più consistente sull'Italia settentrionale, dietro il fronte freddo

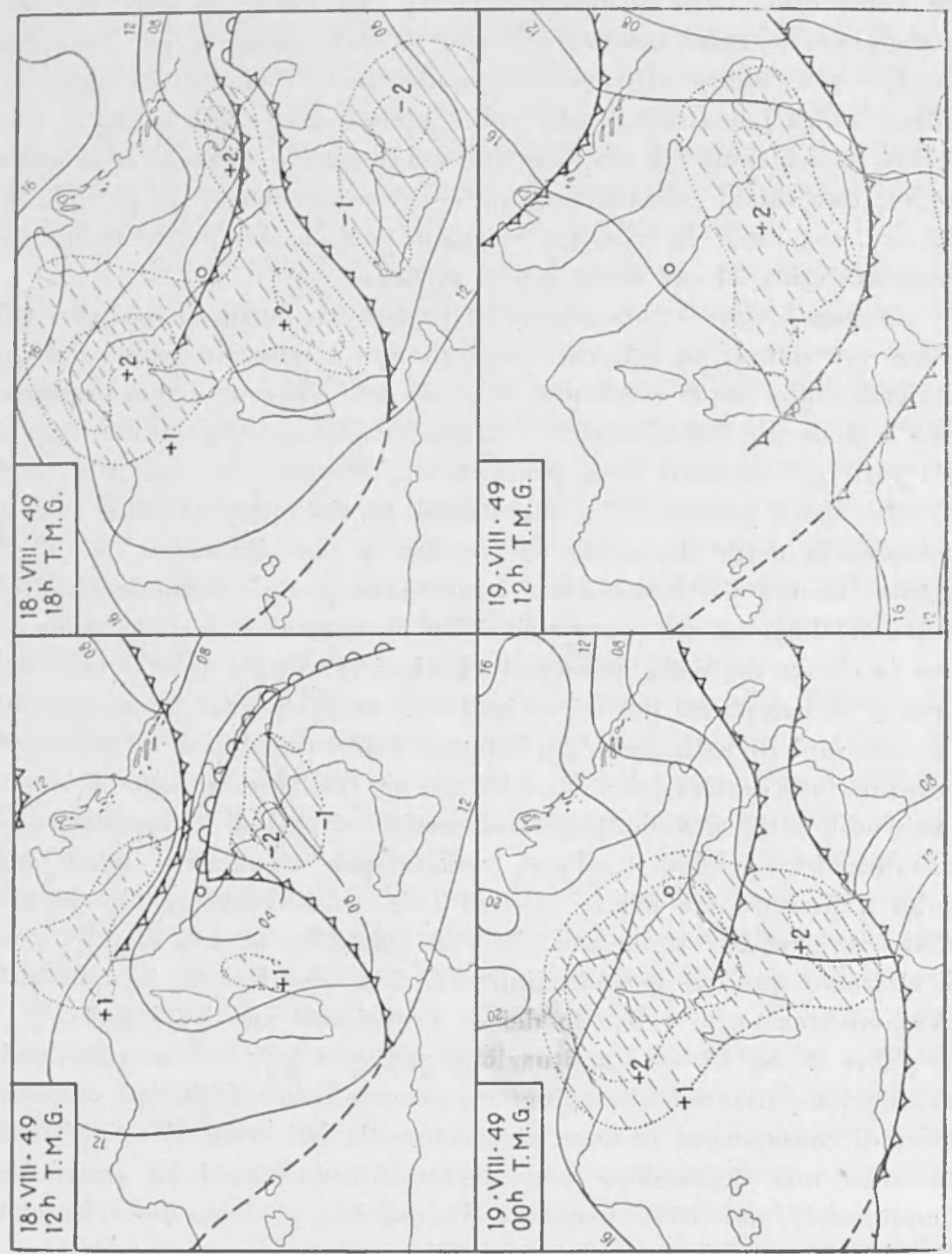

d'origine balcanica che ha incominciato ad interesare il Golfo ligure.

L'attività microsismica denuncia due distinte agitazioni di periodi rispettivamente di $2^{\mathrm{s}}$ ca. e $3 \mathrm{sec}$; è quindi da presumere che l'oscillazione sismica di periodo più hreve, che attualmente ha anche 
un'ampiezza minore, sia da mettere in relazione con il debole nucleo positivo in prossimità della costa sarda, mentre laltra di 3 sec è da collegare con le tendenze positive sul Golfo ligure.

Ore $18 \mathrm{del} 18$.

L'ondulazione sul fronte tropicale si è spostata a E, per cui il fronte stesso viene ad interessare il Tirreno meridionale; mentre il fronte freddo d'origine balcanica continuando il suo movimento verso SO interessa ora il Tirreno centro-settentrionale.

La depressione mediterranea è andata attenuandosi come viene messo in evidenza anche dai nuclei; mentre infatti il negativo sta scomponendo nello Jonio, i nuclei positivi sopra segnalati e che ora si trovano l'uno nel Tirreno sud-occidentale fra la Sardegna e la Sicilia e l'altro sul Tirreno settentrionale sono immersi in una vasta zona positivo. In corrispondenza all'andamento delle tendenze positive l'attività microsismica è in sensibile aumento $\mathrm{ma}$, come si può notare nel grafico che rappresenta l'andamento delle ampiezze, detto aumento è più marcato per l'agitazione di periodo minore, la guale diviene preponderante.

Ore 01 del 19/8. - I due sistemi frontali proseguono nella loro evoluzione; mentre quello tropicale ha quasi oltrepassato il Tirreno, quello di provenienza balcanica sta attraversando ora il Tirreno cen- 
tro-meridionale. A questa ora il nucleo positivo più settentrionale si è esteso verso sud interessando anche il Tirreno centrale. L'attività microsismica nel frattempo ha subito una inversione nella intensità delle due componenti: l'agitazione collegata col nucleo più a sud ha superato la fase massima e va declinando mentre l'agitazione connessa con le perturbazioni di provenienza balcanica prevale nettamente.

Ore $13 \mathrm{del}$ 19/8. - I sistemi frontali sono ormai entrambi fuori del Tirreno; solo il secondo nucleo positivo la cui estensione va diminuendo interessa ancora il Tirreno centro-meridionale. L'agitazione microsismica tende anch'essa a spegnersi.

Settembre 7-8-9-10. — Un fronte fredlo proveniente da NO si riallaccia sul Mediterraneo occidentale intorno alle 06; esso è accompagnato da venti settentrionali piuttosto deholi e camminando a piccola velocità seguito da un nucleo positivo che dopo alcune ore non si rintraccia più sulle coste. Una agitazione microsismica debole si inizia intorno ale ore 10 ca. periodo $4 . \mathrm{sec}$ e scompare alcune ore dopo. E avvenuto evidentemente che il fronte freddo, per cause locali, si è molto attenuato; ma, come spesso avviene, esso non è scomparso e quando le mutate condizioni lo permettono riprende energia e riappare. Infatti lo stesso fronte freddo nelle giornate dell'8/9 investe la Sardegna e poi il Tirreno; poco dopo l'agitazione microsismica riprende, questa volta con periodo assai più lieve.

Nel corso dello spoglio delle registrazioni sismografiche abbiamo notato come una agitazione sorta in concomitanza con una perturbazione atmosferica, pur variando successivamente di ampiezza e di periodo a seconda delle variazioni della energia e della posizione dei nuclei di tendenze positive, mantiene sempre un suo carattere morfologico specifico. Nel caso attuale invece fra l'agitazione di $4^{\mathrm{s}}$ iniziale e quella che riprende intorno alle ore 12 non si nota alcuna rassomiglianza; ciò conferma che il fronte freddo si è rigenerato raggiungendo da $\mathrm{E}$ il Tirreno centrale. La successiva evoluzione segue il corso normale; comunque la concordanza tra agitazione rapida (circa $2^{\text {s) }}$ e perturlazioni vicino Roma è fuori dubbio.

Settembre 30 ed ottobre 1-2. - Si tratta anche qui di un caso molto significativo. Una perturbazione frontale tropicale proveniente dalla Penisola Iberica attraversa da W a E il Mediterraneo centrale continuando il suo processo di occlusione. Preceduto da un nucleo negativo che passando dalla Sardegna alle coste della Penisola si mostra in fase di netto approfondimento, il fronte caldo del sistema entra nel Tirreno la sera del 30 ; il fronte freddo e l'occlusione vi en- 
trano poco prima delle ore 00 dell'1. Il nucleo positivo si presenta poche ore dopo esattamente insieme allo insorgere di una agitazione microsismica molto pura di 3,5 sec che diviene in poche ore assai ampia.

Il nucleo positivo segue una traiettoria alquanto meridionale, dalla Sardegna alle Coste Calabro-Campane, ciò che spiega il periodo leggermente alto; comunque nella sera del giorno 1 il periodo si abhrevia (il nucleo è a $S$ di Ponza) giungendo a 2 sec per poi aumentare di nuovo.

Ottobre 11-12-13. - Dal punto di vista della individuazione della causa è uno dei più signnificativi, poiché nelle carte del tempo compare un nucleo positivo sul Tirreno centro-meridionale apparentemente non proveniente da atre zone, e stazionante a lungo fino ad approfondirsi notevolmente e poi attenuarsi sul posto. Come situazione frontale non si ha che una occlusione in Sicilia, Tirreno meridionale, Calabria e Ionio. I meteorologi analizzatori hanno accennato una rigenerazione frontale sulla occlusione, evidentemente suggerita dalle tendenze positive anzidette; tuttavia non si è formato un settore secondario. L'agitazione microsismica la cui ampiezza varia contemporaneamente e nello stesso senso dell'intensità del nucleo positivo, ha un periodo oscillante tra i 2,5 e i 3 sec.

Ottobre 19-20. - E questa una ripetizione del caso precedente salvo la minore energia in giuoco; anche qui vi è accordo tra agitazione e nucleo positivo sul Tirreno centro-meridionale.

Novembre. - Il novembre è ricco di casi di agitazione microsismica di breve periodo e di grande intensità che si susseguono con brevi interruzionti. La prima di esse è la tempesta microsismica iniziata nelle prime ore del giorno 3 con una agitazione avente periodo di 2 sec che si sovrappone ad una fortissima agitazione di evidente origine atlantica di periodo di $7.8 \mathrm{sec}$; l'agitazione di corto periodo raggiunge la sua massima intensità intorno alle 21 del giorno 3 stesso, poi prosegue nei giorni seguenti sempre sovrapposta all'altra. Il collegamento dell'agitazione di breve periodo con il passaggio di perturbazioni tropicali sul Tirreno ed in particolare con i nuclei positivi che le seguono è fuori discussione; tuttavia se ne omette qui una dimostrazione circostanziata sia perché abbiamo ritenuto di conferire minore importanza agli esempi di agitazione multipla come il presente sia perché tutte le situazioni del novembre offrono una forte analogia tra loro per la zona mediterranea, circostanza questa che ci ha indotto a.descrivere con ogni dettaglio una di esse, quelle dei giorni 
25-28 e a fare semplice accenno delle altre. Un secondo caso di agitazione ad origine tirrenica di grande intensità si verifica immediatamente dopo, nei giorni 7,8 e 9 novembre, ma anche questa sovrapposta ad altra amplissima di periodo di $7-5,5$ sec. Un terzo caso piu modesto per ampiezza e durata ma sempre notevolissimo. si verifica il giorno 11 e 12 ed un quarto nei giorni 15, 16 e 17. Anche per questi tre casi valgono le considerazioni fatte sopra.

Praticamente senza interruzione ma solo con intervalli di maggiore o minore intensità, l'agitazione perdura per tutto il mese, così come la situazione meteorologica sostanzialmente ferma nelle grandi linee rinnova incessantemente il suo ciclo tipico costituito dal passaggio sull'Italia di un nucleo negativo, di una depressione collegata a sistema tropicale in fase di occlusione e del nucleo positivo che conclude la vicenda; è in corrispondenza del passaggio di quest'ultimo elemento che l'attività microsismica si riacutizza. Ciò si ripete ovviamente con variazioni caratteristiche caso per caso nei griorni 19, 20 e 21 e poi ancora nei giorni 23 e 24 e infine nei giorni dal 25 al 28 novemlore.

È quest'ultima situazione che, anche ad illustrazione di tutte le precedenti dello stesso mese di novembre, ablbiamo scelto per una locumentazione più circostanziata.

Novembre 25, ore 18. - Tutta l'Europa in regime di bassa pressione è sede di numerose perturbazioni frontali. Per quanto riguarda il Mediterraneo centrale ed occidentale la situazione è comandata da una depressione piuttosto notevole il cui centro è sulla Tunisia. Il Tirreno e l'Italia sono attraversati da successivi fronti caldi secondari; la direttrice delle correnti è in direzione SN.

Un centro depressionario è in lento movimento verso $\mathrm{NNE}$ e difatti le tendenze barometriche mostrano un nucleo negativo abloastanza attivo sul Tirreno meridionale.

Si hanno venti forti e fortissimisulla Sicilia occidentale e sul basso Tirreno che divengono forti e moderati più a $\mathrm{N}$; piogge intense si hanno sull'Italia settentrionale e parte della centrale. L'agitazione microsismica è ancora praticamente irrilevante.

Alle ore 21 tutto il sistema mediterraneo si è spostato verso NNE mentre il centro depressionario e le perturbazioni frontali si sono trasportate di poco in direzione $N$. La zona di tendenze negative che in precedenza interessava tutto il Tirreno centrale e meridionale ed il canale di Sicilia, si è ora scisso in due nuclei separati: uno, 
il precedente, si è spostato nettamente verso $\mathrm{N}$ investendo l'Italia centro-settentrionale, l'altro si è meglio caratterizzato nel canale di Sicilia; fra i due ha preso consistenza un nucleo positivo. All'interno

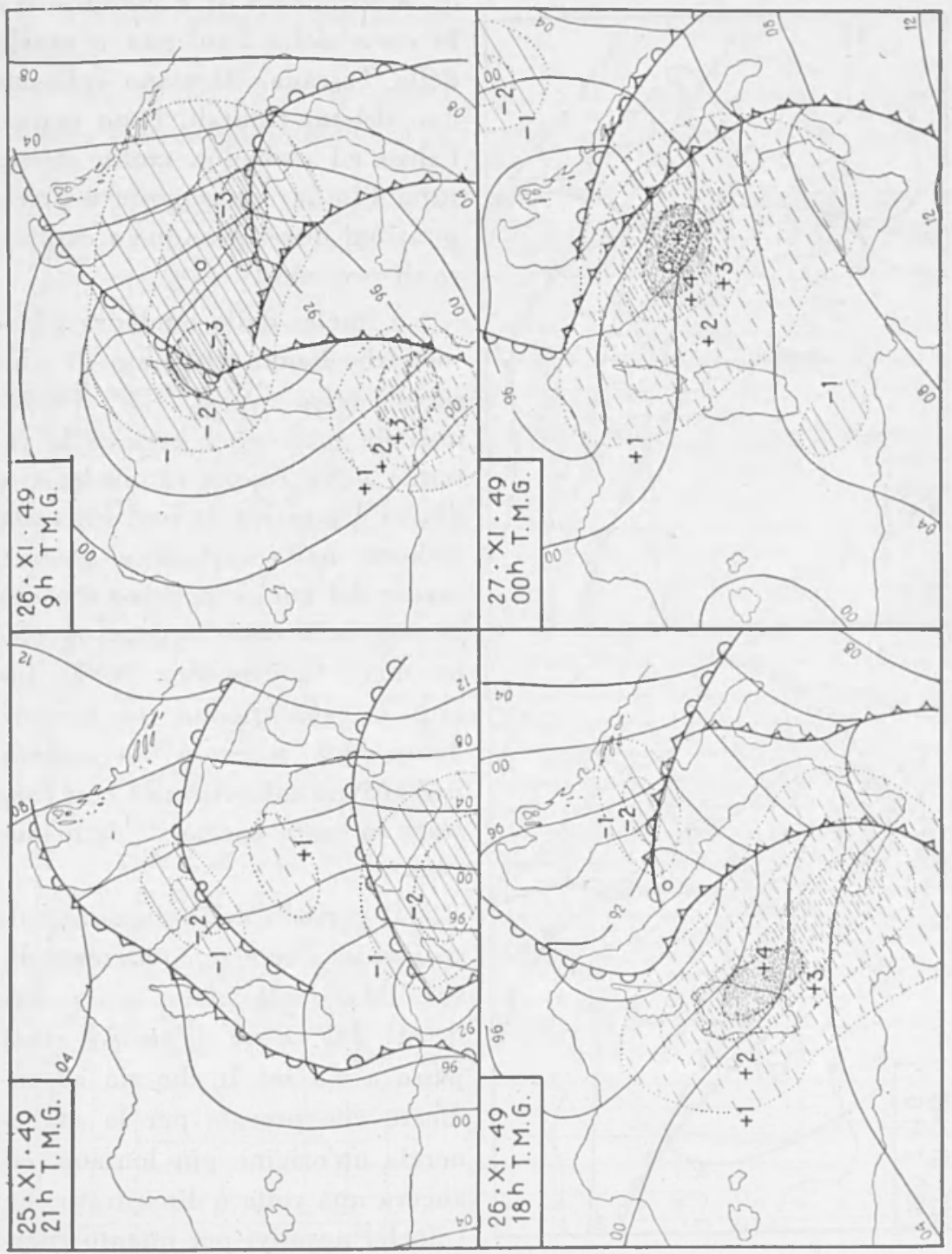

della Tunisia si va delineando un nucleo positivo notevole che successivamente come vedremo avanza verso $\mathrm{N}$.

I venti permangono forti a $S$ e vanno rinforzandosi verso $N$. L'agitazione microsismica va prendendo consistenza con periodo di 
3 sec, in accordo con il nucleo positivo che procede verso $N$ e successivamente raggiunge il continente verso le 00 del 26.

Alle ore 00 del 26 il centro depressionario nel suo spostamento

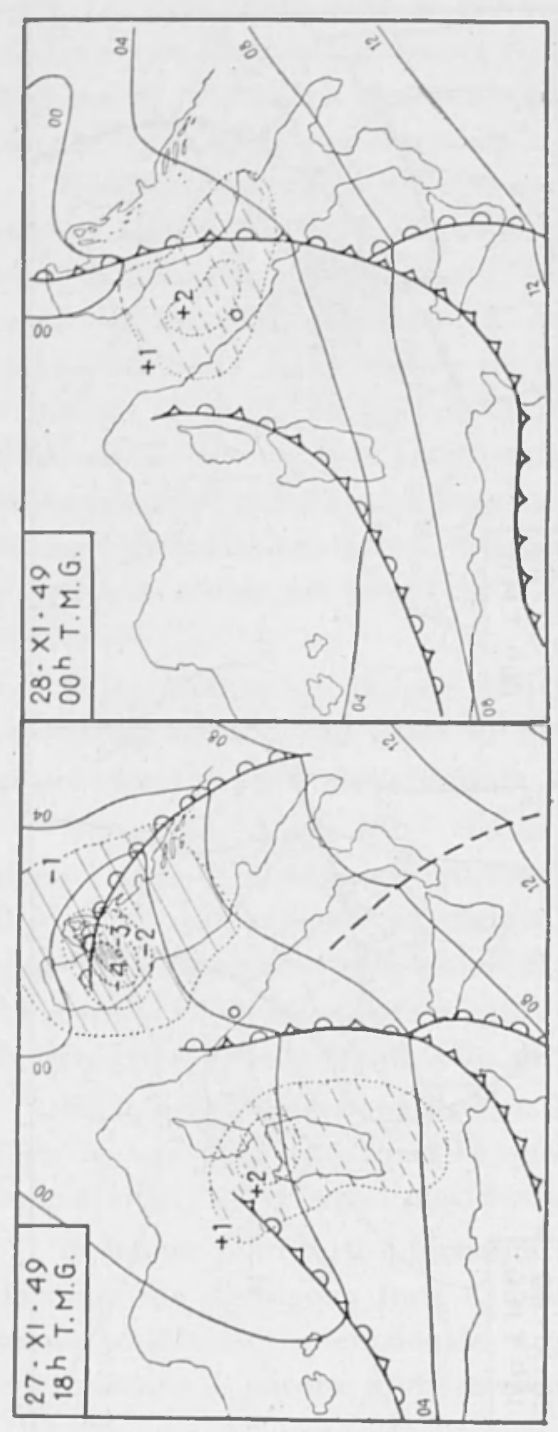
in direzione $N$ si $\dot{e}$ portato tra le coste della Sardegna e quelle della Toscana. Risutano collegati due sistemi frontali, l'uno dentro l'altro ed ambedue molto attivi; tutta l'Italia è sottoposta a precipitazioni quasi ovunque a carattere di rovescio.

I nuclei delle tendenze barometriche hanno viaggiato di conserva verso NNE. Di fondamentale interesse ̀̀ seguire le vicende della coppia di nuclei negativo e positivo di pari intensità indicati nella cartina e specialmente del nucleo positivo che ora avanza dalle coste tunisine in pieno mare in direzione NNE. Infatti è evidentissima la concordanza del notevole incremento dell'attività microsismica con l'entrata in mare aperto di detto nucleo.

ll periodo dell'agitazione microsismica è fondamentalmente diverso da quello delle ore precedenti: dal valore di tre sec circa passa a 4,5 sec il che sta ad indicare che prevale per la sua energia un'origine più lontana, ed ancora una volta è dimostrato che i nuclei negativi per quanto energici e in rapido spostamento non costituiscono una causa efficiente per i microsisni.

I venti permangono forti e lo stato del mare è ovunque molto agitato; l'incremento dell'attività microsismica d'altra parte non può 
dunque mettersi in relazione altro che con la comparsa del nucleo di tendenze positive; inoltre seguendo la variazione dell'intensità dell'attività microsismica misurata in funzione dell'ampiezza in relazione all'intensità del nucleo è facile notarne il concorde andamento.

$\mathrm{Ci}$ riserviamo di comunicare in una prossima nota i risultati quantitativi.

Ore 18 del 25. - Il nucleo positivo si è approfondito e notevolmente esteso continuando a spostarsi in direzione $\mathrm{N}$. II nocciolo

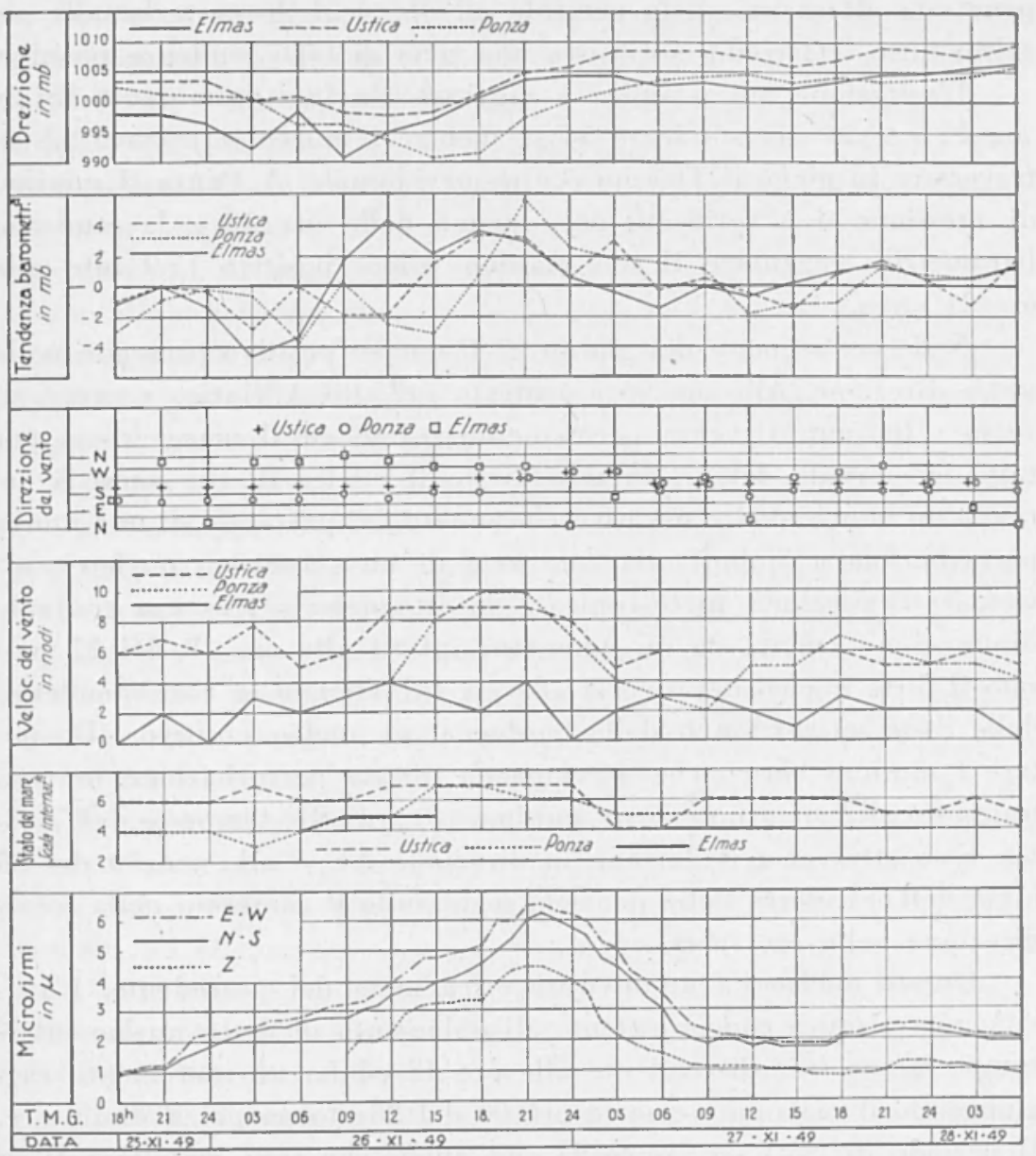

più intenso tende a piegare la sua marcia in direzione $\mathrm{NE}$; cioè dalle coste orientali sarde investe il Tirreno centrale e si dirige verso le coste dell'Italia centrale. Alle ore 18 l'agitazione microsismica entra in una fase parossistica; il periodo è diminuito ora a 4 sec circa. 
La diminuzione del periodo rispetto alle ore precerlenti, nonostante il fortissimo aumento dell'ampiezza è particolarmente significativo e prova l'avvicinamento della origine dei microsismi. Anche in questo caso, in maniera ancora più evidente, l'unica causa efficiente a cui si possa attribuire la sorgente di energia della eccezionale attività microsismica è il considerevole nucleo di tendenze positive in mare aperto.

Alle ore 00 del 27 la zona più profonda del nucleo positivo è penetrata all'interno della penisola all'altezza delle coste laziali; peraltro tutto il Tirreno costituisce una zona di forti tendenze positive.

L'agitazione microsismica a raggiunto la fase culminante fra le ore 21 e e 23 con ampiezze 6-7 u, mentre il nuceo si trovava ad attraversare in pieno il Tirreno centro-meridionale. A Ponza il minimo di pressione si è verificato poco prima delle ore 18 e la tendenza barometrica raggiunge il suo masimo valore positivo $(5,9 \mathrm{mb})$ alle ore 21 circa.

Nelle ore seguenti del giorno 27 il nucleo positivo prosegue nella stessa direzione. Alle ore 06 è centrato sull'alto Adriatico e prosegue verso i Balcani. Il vento permane fortissimo ed il mare grosso per tutta la giornata del 27. Intanto dietro il nucleo di cui sopra si accenna un nuovo nucleo negativo che prelude al passaggio di una nuova perturbazione e quindi alla comparsa di un successivo nucleo positivo. Nell'agitazione microsismica è da attendersi perciò una graduale diminuzione seguita da un aumento; infatti alle ore 18 del 27 passato il forte nucleo negativo si aflaccia sul Tirreno in corrispondenza delle coste settentrionali della Sardegna un nucleo positivo. (Da notare i minimo barico che accompagna questa perturbazione in contrapposto alla mancanza di un minimo barico nelle tempeste dell'aprile). Esso attraversa il Tirreno in direzione NE e alle ore 00 del 28 è già nell'entroterra della penisola seguitando il cammino nella stessa direzione nelle ore successive.

Questo nucleo ha un'intensità circa metà del precedente. L'attività microsismica concordemente all'andamento di detto nucleo interrompe la sua fase discendente alle ore 18 ed ha un incremento raggriungendo il massimo verso le ore 00 del 28 ; torna poi a diminuire. II periodo di $2,5-3$ sec concorda con quelli che sono stati trovati in occasione di tempeste microsismiche provenienti, come era stato presunto, dalla stessa zona.

Dicembre 2-3-4. - Alle ore 12 del giorno 2 un settore tropicale si affaccia sul Tirreno preceduto da un nucleo negativo ben definito 
che ha cominciato a manifestarsi alcune ore prima. Le registrazioni sismografiche non segnalano nulla di notevole. Alle ore 06 del 3 compare un nucleo positivo netto sul golfo di Lione, che alle ore 12 passa sulla Liguria e sulla Sardegna ed alle ore 18 sul Tirreno centrale; a questa stessa ora il settore tropicale interessa la Sicilia e lo Ionio.

L'agitazione microsismica comincia nella mattinata del giorno 3 e segue e vicende del nucleo positivo.

Dicembre 8-9-10-11. - È un caso di agitazione particolarmente ampia composta di due periodi molto distanziati (l'uno di $7 \mathrm{sec}$, l'altro di 3 sec) e ben distinti fra loro. 11 perioro lungo è chiaramente collegato ad una profonda depressione atlantica che interessa tutta l'Furopa occidentale e settentrionale, mentre il periodo breve, che prevale come ampiezza sull'altro solo dale prime ore del giorno 10 in poi, è dovuto ad una perturbazione frontale che entra nel Tirreno intorno alle ore 18 del giorno 9 . Tale perturbazione è collegata ad una coppia di nuclei negativo-positivo assai marcati provenienti dal NW; ancora una volta l'andamento dell'agitazione microsismica è in accordo con lo spostamento del nucleo positivo.

Dicembre 16-17-18. - Tutta l'Europa centro-occidentale è in regime di bassa pressione, con tendenza, il giorno 16 , alla formazione di un minimo secondario sull'Italia settentrionale; tale minimo peraltro va spostandosi piuttosto velocemente verso SE. Sulla regione Mediterranea sono presenti vari fronti a carattere freddo fra loro parolleli che si inseguono camminando verso ESE. L'agitazione microsismica è assai ampia e varia con insolita rapidità i suoi caratteri morfologici ed il suo periodo oscillante fra i 2 ed i 4 sec. Si può effettivamente stabilire una concordanza con sfasamento nel tempo fra le variazioni dell'agitazione microsismica ed il passaggio dei fronti freddi, ma è difficile ricostruire dalle osservazioni l'entità e l'ubicazione dei nuclei positivi seguenti ciascun fronte: ad ogni modo l'esistenza nella zona di tali nuclei è fuori discussione. Peraltro la distribuzione minuta delle tendenze positive e negative è durante la giornata del 17 travolta dall'arrivo di un poderoso nucleo positivo proveniente dalla Francia. È con l'arrivo di tale nucleo sul Tirreno che l'agitazione, già ampia, diviene intensissima con periodo prevalente sui 3,5 sec. Per tutta la durata della perturbazione i venti si son mantenuti deboli o moderati.

4. Conclusione. - Da quanto sopra esposto, che è quanto dire dall'esame comparato tra il comportamento dell'attività microsismica 
e la contemporanea evoluzione delle perturbazioni atmosferiche, condotto con continuità per la durata di un anno servendoci di tutti i mezzi ed i metodi offertici dalla odierna organizzazione dei servizi meteorologici risulta provato che:

a) il ritardo che costantemente si osserva tra il passaggio di un centro depressionario, quando esiste, ed il sorgere di una attività microsismica sta a confermare che questa non dipende da esso;

b) non sussiste diretta dipendenza tra il passaggio di fronti a carattere fredllo ed attività microsismica;

c) non esiste dipendenza alcuna dell'attività microsismica con fronti a carattere caldo;

d) infine non esiste alcuna correlazione costante tra forza del vento, direzione del vento, stato del mare da una parte ed attività microsismica dall'altra;

e) la quasi totalità dei casi esaminati ha invece indicato in modo assolutamente evidente la dipendenza diretta dell'attività micro. sismica dalla presenza, estensione ed intensità dei nuclei di tendenza barometrica positiva in mare aperto; i pochi restanti casi in cui questa evidenza non è apparsa così certa ed esclusiva purtuttavia non costituiscono prova contraria; è importante notare infatti che anche in questi casi tale elemento è sempre esistito anche se non presentava una apparente netta prevalenza rispetto agli altri elementi. In altre parole detto elemento è stato sempre presente e solo in qualche caso le osservazioni non hanno permesso una determinazione causale più precisa ed univoca.

Il risultato fondamentale di questa ricerca, che fu già oggetto di una nostra comunicazione alla XLII Riunione della Società Italiana per il Progresso delle Scienze (Roma, novembre 1949), ̀̀ pertanto il seguente: $i$ microsismi di origine mediterranea vengono generati nelle zone in mare aperto interessate da nuclei di tendenza barometrica positiva, stazionari o in movimento.

Una volta individuata la causa specifica abbiamo potuto inoltre provare in maniera molto più esauriente e diretta, di quanto ai vari ricercatori fino ad ora non era stato possibile, che il periodo iniziale della agitazione microsismica viene determinato dal luogo ove ebbe origine l'agi'azione stessa e rappresenta un elemento fondamentale caratteristico legato alle modalità della generazione dell'oscillazione microsismica, alla intensità del nucleo nonché alla profondità delle acque marine e delle caratteristiche sismo-elastiche e di struttura della crosta terrestre. Detto periodo subisce poi variazioni dello stesso senso 
delle variazioni sia della distanza sia della intensità dell'agitazione; ed inoltre una agitazione sorta in concomitanza con una perturbazione atmosferica, pur variando successivamente di ampiezza e di periodo a seconda delle variazioni della energia e delle posizioni dei nuclei di tendenze positive, mantiene sempre un suo carattere morfologico specifico.

Questi risultati che, per motivi impostici dallo scopo della nostra attuale comunicazione, diamo ora solo in forma qualitativa sono oggetto di studi da noi condotti al fine di trarre elementi quantitativi circostanziati in riguardo sia all'entità dei valori delle grandezze fisiche in giuoco sia sopratutto nei riguardi delle strutture delle variazioni barometriche a cui deve imputarsi il fenomeno dell'agitazione microsismica. Un particolare scopo delle ricerche in corso è quello di studiare le modalità con cui vengono generate le oscilazione elastiche terrestri e quindi la trasmissione di energia dall'atmosfera alla crosta terrestre. Un primo esame basato sulle risultanze del presente studio, come già si è rilevato nel corso dell'esposizione, ci conduce a ritenere che più che l'effetto dinamico del vento, come generalmente si è indotti a pensare, le oscillazioni vengono generate da impulsi di pressione provocati presumibilmente dalla tumultuosa sostituzione in quota di masse di aria più dense a masse di aria meno dense.

Una risposta chiarificatrice a questi problemi potrà aversi prendendo in esame, oltre agli elementi già considerati. lo stato di turbolenza in quota.

Questo lavoro è stato eseguito in collaborazione col Servizio Meteorologico dell'Areonautica Italiana.

$$
\text { Roma - Istituto Nazionale di Geofisica - Marzo } 1950 .
$$

\section{RIASSUNTO}

Questo studio fa parte di una ricerca sistematica sui microsismi in relazione alle perturbazioni atmosferiche intrapresa dall'Istituto Nazionale di Geofisica in collaborazione col Servizio Meteorologico dell'Aeronautica Italiana, ed è lo sviluppo documentato di una indagine eseguita dagli Autori $i$ cui risultati furono oggetto di una comunicazione alla XLII Riunione della Società Italiana per il Progresso delle Scienze. (Roma, novembre 1949).

Tra $i$ principali problemi già indicati dagli Autori in precedenti 
lavori viene qui affrontato e risolto quello fondamentale della ricerca delle cause dirette che generano i microsismi. Dopo una messa a punto delle conoscenze acquisite nei riguardi di detto problema ed una critica dei metodi usati nei tentativi fino ad ora fatti per risolvere questa annosa questione si dimostra che la sola via possibile da intraprendere per risolvere il problema stesso in maniera decisiva ̀े quella di eseguire una indagine meteorologica adeguata. Vengono quindi esposti il criterio base che ha diretto la presente ricerca nonché $i$ mezzi ed $i$ metodi adoperati che hanno consentito agli Autori di pervenire ad un risultato oggettivo indipendente da qualsiasi ipotesi aprioristica.

Mediante un esame comparato del comportamento dell'attività microsismica e della contemporanea evoluzione delle vicende meteorologiche considerate nel complesso unitario dei loro elementi costitutivi, condotto con continuità per la durata di un intero anno, gli autori hanno ottenuto, in maniera precisa ed univoca, il seguente risultato fondamentale di assoluta evidenza: " $i$ microsismi di origine mediterranea vengono generati nelle zone in mare aperto interessate da nuclei di tendenza barometrica positiva, stazionari o in movimento $\gg$.

Inoltre un'agitazione microsismica sorta in concomitanza con una perturbazione atmosferica, pur variando successivamente di ampiezza e di periodo a seconda delle variazioni della energia e della posizione dei nuclei di tendenza positiva, mantiene sempre un suo carattere morfologico specifico.

Gli Autori ritengono che le oscillazioni microsismiche vengono generate da impulsi di pressione provocati presumibilmente dallo stato di turbolenza in quota.

\section{BIBLIOGRAFIA}

(1) Gıng M.: Su alcuni aspetti caratteristici dei microsismi a Roma in relazione con fattori meteorologici. Annali di Geofisica 2, 24-39, n. 1 (1949).

(2) Rosını E.: L'agitazione microsismica e le perturbazioni atmosferiche. Rivista di Meteorologia Aeronautica, a. VIII, n. 4, 30-40 (1948).

(3) Bibliography on microseisms. California Institute of Technology, Pasadena 1949.

(4) De Rossı M. S.: Boll. del Vulcanismo italiano, 1 (1784).

(5) Algù̇ J.: Relation entre quelques mouvements microséismique et l'existence, le position et la distance des cyclones a Manille. Procès verbaux et memoires du Congrès international de Meteorologie, Paris 1900. 
(6) Wiechert E.: Discussion, Verh. der Zweiten Internat. Seism. Konf. 1905 Gerlands Beitr. Geophysik, 2, 41.42.

(7) Gutenberg B.: Die seismiche Bodenunruhe. Diss. Gottingen, Gerlands Beitr. z. Geophysik, 11, 314-353 (1912)

(8) BeNndonf II.:Uber die microseismischen Bewegungen. Sonderabdruck aus Geologische Rundschau, 1, 183-186 (1910).

(9) НескеR O.: Ergebnisse der Beobachtung der mikroseismischen Bewegungen an den europäischen stationen wührend des Winters 1912.13. Mitt. Zentr. Int. Seism. Ass. Gerlands Beitr. z. Geophysik 14, 1-13 (1914).

(10) Gherzı E.: Cyclones and microseisme. Gerlands Beitr. z. Geophysik 36, 2023 (1932).

(11) BanerJi S. K.: Theory of microseisms. Proc. Ind. Acad. Sc. 1, 727-753, n. 10 (1935).

(12) Ramirez J. E.: An experimental investigation of the nature and origin of microseisms at S. Louis Missouri. Bull. Seism. Soc. Am. 30, 35-84; 139-178 (1940).

(13) Gilmore M. H.: Microseisms and ocean storms. Bull. Seism. Soc. Am. 36, 89-119 (1946).

(14) LeEt, L. Don: Microseisms in New England - case history of a storm. Geophysics 12, 639-650 (1947).

- -: Microseisms in New England-case history H. Bull. Seism. Soc. Am. $38,173-178$, n. 3 (1948).

(15) Jones W. M.: New Zealand microseisms and their relation to weather conditions. $7^{\text {th }}$ Pac. Sci. Congress (1949).

(16) Atti della Soc. It. per il Progresso delle Scienze, Roma. Riunione XIII, 1949. 